\title{
A SURVEY OF C*-ALGEBRAIC QUANTUM GROUPS, PART II
}

\author{
Johan Kustermans \& Lars Tuset
}

In part I of this survey [29], we introduced the reader to the subject of quantum groups from the $\mathrm{C}^{*}$-algebra point of view and surveyed Woronowicz's theory of compact quantum groups.

In this part, we begin by considering the category of multiplier Hopf *-algebras introduced by A. Van Daele. It is a self-dual category which contains both compact and discrete quantum groups. The Pontryagin Duality Theorem is a particular case of this duality. The dual of a compact quantum group is a discrete quantum group and vice versa.

In the final section we briefly consider the theory of general locally compact quantum groups. We state the recently established definition of a locally compact quantum group given by S. Vaas and the first author. The subject is rather technical for a number of reasons and involves unbounded operators, multiplier algebras and weights. We briefly look into the quantum group version of the motion group $E(2)$ of the plane.

We devote a section to show how $C^{*}$-algebraic quantum groups are related to the examples of quantum groups studied by V. G. Drinfeld and his collaborators. Those are obtained as deformations or quantizations of universal enveloping algebras of Lie algebras. We explain what is meant by analytic and formal quantization, and demonstrate how quantum $S U(2)$ can be obtained as a quantization of the standard Poisson structure on $S U(2)$. The tensor $\mathrm{C}^{*}$-category of *-representations of the quantized universal enveloping algebras of Drinfeld forms the link to the compact quantum groups of Woronowicz. We outline the theory of $R$-matrices and QYBE-equations. The section ends with a discussion of the quantum-plane approach of Y. Manin and some remarks on the differential structure of quantum Lie groups. 
Throughout this paper we use the symbol $\odot$ to denote an algebraic tensor product and $\otimes$ to denote its topological completion with respect to the minimal tensor-product norm. The only exceptions are made when we discuss $h$-adic completions in Section 6 .

\section{Table of contents}

5. Multiplier Hopf *-algebras

6. The Drinfeld Approach to Quantum Groups

7. Locally Compact Quantum Groups

\section{Multiplier Hopf *-algebras}

In this section we enlarge the category of compact quantum groups to a category that also includes discrete quantum groups. Woronowicz and P. Podleś ([42]) defined discrete quantum groups as duals of compact ones. On the other hand, E. Effros \& Z.J. Ruan ([13]) and Van Daele ([62]) introduced discrete quantum groups without basing the definition on the theory of compact quantum groups. In [61], Van Daele investigated multiplier Hopf *-algebras possessing a left-invariant Haar functional. His framework is purely algebraic but is broad enough to contain both the compact and the discrete quantum groups. Moreover, his category is closed under the formation of duals and the quantum doubles of Drinfeld (and more generally, forming bi-crossed products). We have chosen to work in this setting because it allows us to give a good intuitive picture of quantum groups without having to resort to the heavy $\mathrm{C}^{*}$-algebraic machinery used in the general theory of locally compact quantum groups.

Suppose $A$ and $B$ are non-degenerate *-algebras and consider a multiplicative linear mapping $\pi$ from $A$ to $M(B)$. We say that $\pi$ is non-degenerate if the vector spaces $\pi(A) B$ and $B \pi(A)$ are both equal to $B$. Such a map has a unique multiplicative linear extension to $M(A)$, which we denote by the same symbol as used for the original mapping. Of course, we have similar definitions and results for anti-multiplicative mappings. When we work in an 
algebraic setting, we will always use this form of non-degeneracy, as opposed to the non-degeneracy of *-homomorphisms between $\mathrm{C}^{*}$-algebras defined earlier. Given a linear functional $\omega$ on a nondegenerate *-algebra $A$ and an element $a \in M(A)$, we define the linear functionals $\omega a$ and $a \omega$ on $A$ by setting $(a \omega)(x)=\omega(x a)$ and $(\omega a)(x)=\omega(a x)$ for every $x \in A$. More information about non-degenerate algebras can be found in the appendix of [64].

Now let $\omega$ be a linear functional on a ${ }^{*}$-algebra $A$. Then:

1. $\omega$ is called positive if $\omega\left(a^{*} a\right) \geq 0$ for every $a \in A$.

2. If $\omega$ is positive, then $\omega$ is called faithful if, for every $a \in A$, we have $\omega\left(a^{*} a\right)=0 \Rightarrow a=0$.

Definition 5.1 We call $(A, \Delta)$ a multiplier Hopf ${ }^{*}$-algebra if $A$ is a non-degenerate *-algebra and $\Delta$ is a non-degenerate *homomorphism from $A$ into $M(A \odot A)$ such that:

1. $(\Delta \odot \iota) \Delta=(\iota \odot \Delta) \Delta$.

2. The unique linear maps $T_{1}, T_{2}$ from $A \odot A$ into $M(A \odot A)$ such that

$$
T_{1}(a \otimes b)=\Delta(a)(b \otimes 1) \quad \text { and } \quad T_{2}(a \otimes b)=\Delta(a)(1 \otimes b)
$$

for all $a, b \in A$, are bijections from $A \odot A$ onto $A \odot A$.

In [64] Van Daele proves the existence of a unique nonzero *-homomorphism $\varepsilon$ from $A$ to $\mathbb{C}$ such that $(\varepsilon \odot \iota) \Delta=(\iota \odot$ $\varepsilon) \Delta=\iota$. Furthermore, he proves the existence of a unique antiautomorphism $S$ on $A$ such that

$m(S \odot \iota)(\Delta(a)(1 \otimes b))=\varepsilon(a) b \quad$ and $\quad m(\iota \odot S)((b \otimes 1) \Delta(a))=\varepsilon(a) b$

for all $a, b \in A$. Here $m$ denotes the linear map from $A \odot A$ to $A$ induced by the multiplication on $A$, and, as expected, $\varepsilon$ is called the co-unit and $S$ the antipode of $(A, \Delta)$. Moreover, we have $S\left(S\left(a^{*}\right)^{*}\right)=a$ for all $a \in A$, and $\sigma(S \odot S) \Delta=\Delta S$, where $\sigma$ denotes the flip-automorphism on $A \odot A$. 
Let $\omega$ be a linear functional on $A$. We say that $\omega$ is leftinvariant (with respect to $(A, \Delta))$ if $(\iota \odot \omega)(\Delta(a)(b \otimes 1))=\omega(a) b$ for all $a, b \in A$. Right invariance is defined similarly.

Definition 5.2 We call $(A, \Delta)$ an algebraic quantum group if it is a multiplier Hopf ${ }^{*}$-algebra with a non-zero positive linear functional $\varphi$ on $A$ that is left-invariant.

The terminology 'algebraic quantum group' should not be confused with 'quantum algebraic group' which could be understood as a quantization of an algebraic group.

In the rest of this section we fix an algebraic quantum group $(A, \Delta)$ and a non-zero left-invariant positive linear functional $\varphi$ on it.

An important feature of $(A, \Delta)$ is the faithfulness and unicity of left-invariant functionals:

1. If $\omega$ is another left-invariant linear functional on $A$, there exists a unique number $c \in \mathbb{C}$ such that $\omega=c \varphi$.

2. The functional $\varphi$ is faithful.

It is clear that $\varphi S$ is a non-zero right-invariant linear functional on $A$, but in general it is not known whether $\varphi S$ is positive. However, one may prove that a non-zero positive right-invariant linear functional on $A$ exists that is unique up to a scalar multiple.

Another important property is the existence of a unique automorphism $\rho$ of $A$ such that $\varphi(a b)=\varphi(b \rho(a))$ for all $a, b \in A$. We call this the weak KMS-property of $\varphi$. Moreover, we have $\Delta \rho=\left(S^{2} \odot \rho\right) \Delta$ and $\rho\left(\rho\left(a^{*}\right)^{*}\right)=a$ for every $a \in A$.

It is possible to introduce a modular function for algebraic quantum groups. It is an invertible element $\delta$ in $M(A)$ such that $(\varphi \odot \iota)(\Delta(a)(1 \otimes b))=\varphi(a) \delta b$ for every $a, b \in A$.

This modular function is, as in the classical group case, a 1-dimensional (generally unbounded) co-representation:

$$
\Delta(\delta)=\delta \odot \delta \quad \varepsilon(\delta)=1 \quad S(\delta)=\delta^{-1} .
$$


As in the classical case, we can relate our left-invariant functional to our right-invariant functional via the modular function: that is, for every $a \in A$, we have

$$
\varphi(S(a))=\varphi(a \delta) .
$$

The following property says, loosely speaking, that every element of $A$ has compact support: Let $a_{1}, \ldots, a_{n} \in A$. There exists an element $c$ in $A$ such that $c a_{i}=a_{i} c=a_{i}$ for every $i \in$ $\{1, \ldots, n\}$.

We move on to discuss duality within the category of algebraic quantum groups:

Let us define a subspace $\hat{A}$ of $A^{\prime}$ by

$$
\hat{A}=\{\varphi a \mid a \in A\}=\{a \varphi \mid a \in A\} .
$$

As in the theory of Hopf ${ }^{*}$-algebras, $\hat{A}$ is a non-degenerate *-algebra:

1. For every $\omega_{1}, \omega_{2} \in \hat{A}$ and $a \in A$, we have $\left(\omega_{1} \omega_{2}\right)(a)=$ $\left(\omega_{1} \odot \omega_{2}\right)(\Delta(a))$.

2. For every $\omega \in \hat{A}$ and $a \in A$, define $\omega^{*}(a)=\overline{\omega\left(S(a)^{*}\right)}$.

We remark that it requires some arguments to show that one gets a well-defined product and ${ }^{*}$-operation on $A$ this way.

The co-multiplication $\hat{\Delta}$ is defined on $\hat{A}$ by $\hat{\Delta}(\omega)(x \otimes y)=$ $\omega(y x)$ for every $\omega \in \hat{A}$ and $x, y \in A$. For this to make sense, $M(A \odot A)$ should be embedded in $(A \odot A)^{\prime}$ in a proper way. A definition of the co-multiplication $\hat{\Delta}$ that does not use such an embedding can be found in Definition 4.4 of [63]. Hence, $(\hat{A}, \hat{\Delta})$ is a multiplier Hopf *-algebra. The co-unit $\hat{\varepsilon}$ and the antipode $\hat{S}$ are defined by:

1. $\hat{\varepsilon}(\omega)=\omega(1)$ for every $\omega \in \hat{A}$.

2. $\hat{S}(\omega)(a)=\omega\left(S^{-1}(a)\right)$ for every $\omega \in \hat{A}$ and $a \in A$. 
Define $\hat{a}=a \varphi \in \hat{A}$ for $a \in A$. The map $A \rightarrow \hat{A}, a \mapsto \hat{a}$ is a bijection. It is the Fourier transform.

Next define the linear functional $\hat{\varphi}$ on $\hat{A}$ by setting $\hat{\varphi}(\hat{a})=$ $\varepsilon(a)$ for every $a \in A$. It is possible to prove that $\hat{\varphi}$ is left-invariant. Furthermore, $\hat{\varphi}\left(\hat{a}^{*} \hat{a}\right)=\varphi\left(a^{*} a\right)$ for every $a \in A$. This implies that $\hat{\varphi}$ is a non-zero positive left-invariant linear functional on $\hat{A}$. Hence the dual $(\hat{A}, \hat{\Delta})$ is an algebraic quantum group.

The algebraic quantum group version of Pontryagin's duality theorem takes the following form:

Theorem 5.3 Let $(A, \Delta)$ be an algebraic quantum group. Then the double dual $(\hat{\hat{A}}, \hat{\hat{\Delta}})$ is an algebraic quantum group isomorphic to $(A, \Delta)$. More precisely, there exists a canonical ${ }^{*}$-isomorphism $\theta: A \rightarrow \hat{\hat{A}}$ such that $\theta(a)(\omega)=\omega(a)$ for all $a \in A$ and $\omega \in \hat{A}$ and that satisfies the equation $(\theta \otimes \theta) \Delta=\hat{\Delta} \theta$.

Using the left Haar functional $\varphi$, one can construct a GNSpair $(H, \Lambda)$ in the usual manner. So $H$ is a Hilbert space and $\Lambda$ is a linear map from $A$ into $H$ such that $\Lambda(A)$ is dense in $H$ and $\langle\Lambda(a), \Lambda(b)\rangle=\varphi\left(b^{*} a\right)$ for all $a, b \in A$. Also, there exists a ${ }^{*}$-representation $\pi: A \rightarrow B(H)$ such that $\pi(x) \Lambda(a)=\Lambda(x a)$ for all $x, a \in A$. Clearly the closure $A_{r}$ of $\pi(A)$ in $B(H)$ is a $\mathrm{C}^{*}-$ subalgebra of $B(H)$. Many notions defined on $A$ (such as the co-multiplication $\Delta$, the functional $\varphi$, the modular function $\delta$, etc.) have 'continuous' extensions to the enveloping $\mathrm{C}^{*}$-algebra $A_{r}$, but these constructions require non-trivial $\mathrm{C}^{*}$-algebra theory (see [28]). We denote this analytic extension of $(A, \Delta)$ by $\left(A_{r}, \Delta_{r}\right)$.

Definition 5.4 An algebraic quantum group $(A, \Delta)$ is:

- of compact type if $A$ is unital,

- of discrete type if there exists a non-zero element $h \in A$ satisfying $a h=h a=\varepsilon(a) h$ for all $a \in A$.

Proposition 5.5 Let $(A, \Delta)$ be an algebraic quantum group. 
- If it is of compact type, its analytic extension $\left(A_{r}, \Delta_{r}\right)$ is a compact quantum group.

- If it is of discrete type, its analytic extension $\left(A_{r}, \Delta_{r}\right)$ is a discrete quantum group in the sense of Woronowicz and Van Daele, i.e.

1. There exists a family of finite-dimensional Hilbert spaces $\left(H_{i}\right)_{i \in I}$ such that $A_{r}$ is ${ }^{*}$-isomorphic to $\sum_{i \in I} B\left(H_{i}\right)$.

2. $\Delta_{r}$ is a non-degenerate ${ }^{*}$-homomorphism from $A_{r}$ to $M\left(A_{r} \otimes A_{r}\right)$ satisfying $\left(\Delta_{r} \otimes \iota\right) \Delta_{r}=\left(\iota \otimes \Delta_{r}\right) \Delta_{r}$.

3. The unique linear maps $T_{1}, T_{2}$ from $A_{r} \odot A_{r}$ into $M\left(A_{r} \otimes A_{r}\right)$ such that

$$
T_{1}(a \otimes b)=\Delta(a)(b \otimes 1) \quad \text { and } \quad T_{2}(a \otimes b)=\Delta(a)(1 \otimes b)
$$

for all $a, b \in A_{r}$, are injective and have dense range in $A_{r} \otimes$ $A_{r}$.

Suppose that $\left(A_{r}, \Delta_{r}\right)$ is a discrete quantum group with $A_{r}$ commutative. Then every $B\left(H_{i}\right)$ has to be commutative and hence 1-dimensional. Thus, $A_{r}$ is isomorphic to $\mathrm{C}_{0}(I)$ with the discrete topology on $I$. The co-multiplication $\Delta_{r}$ induces a multiplication on $I$ turning it into a discrete group.

That discrete and compact quantum groups are dual to each other, can be stated precisely in the following manner.

Proposition 5.6 An algebraic quantum group $(A, \Delta)$ is of compact type if and only if its dual $(\hat{A}, \hat{\Delta})$ is of discrete type.

\section{The Drinfeld Approach to Quantum Groups}

In this section we outline the program initiated and developed by Drinfeld and his collaborators and indicate how their work is related to the $\mathrm{C}^{*}$-algebraic approach to quantum groups. They work with Lie algebras, their universal envelopes and deformations of these, whereas the $\mathrm{C}^{*}$-approach to the subject takes place on the dual side, where Haar weights are defined most naturally and 
where the unboundedness of differential operators does not pose an immediate difficulty.

For a non-compact locally compact matrix group, however, even the coordinate functions on the group cannot all be bounded. This creates severe difficulties in treating examples of locally compact quantum groups, because, as we shall see, quantization is carried out most conveniently when working with coordinate functions, which yield non-commuting 'coordinate function'-generators with nice formulas for the co-multiplication. The $\mathrm{C}^{*}$-algebra of the quantum group is customarily formed by taking bounded functions of these 'coordinate function'-generators in such a way that the the 'coordinate function'-generators are affiliated to the $\mathrm{C}^{*}$-algebra, [75],[74]. The co-multiplication for these new bounded generators is usually given in terms of formulas involving (horrendous) infinite sums. We will define locally compact quantum groups in the next section. In this section quantum $S U(2)$ will be our guiding example.

We start with the concept of quantization. It appears to be the only established way to generate examples of quantum groups that are not groups, including for instance, quantum $S U(2)$. The notion resembles the canonical quantization procedure of obtaining quantum mechanics from classical mechanics, [8],[2],[19]. The standard Poisson bracket $\{\cdot, \cdot\}$ on the commutative *-algebra $C^{\infty}\left(\mathbb{R}^{d} \oplus \mathbb{R}^{d}\right)$ of smooth functions on the classical phase space $\mathbb{R}^{d} \oplus \mathbb{R}^{d}$ is replaced by a commutator $[\cdot, \cdot]$ of self-adjoint unbounded operators representing observables in this new quantum mechanics. The non-commutativity of the operators explains experiments involving the measure processes on the atomic level and implies new fundamental phenomena such as Heisenberg's Uncertainty Principle. Weyl, Moyal, von Neumann and others recognized (via the Weyl transform) this replacement of functions on the phase space by operators, as a deformation of the pointwise product on $C^{\infty}\left(\mathbb{R}^{d} \oplus \mathbb{R}^{d}\right)$, but now extended to an algebra over the ring $\mathbb{C}[[\hbar]]$ of formal power series in a variable $\hbar$ with coefficients in $\mathbb{C}$, [66]. Planck's constant $\hbar$, which is so small that quantum effects are negligible in the classical regime (among physicists this 
is known as Bohr's correspondence principle), is thought of as the deformation parameter. The usual commutative product is recovered when $\hbar=0$.

Recall that $\mathbb{R}^{d} \oplus \mathbb{R}^{d}$ is a symplectic manifold with the standard bilinear form $\{\cdot, \cdot\}$ defined on $C^{\infty}\left(\mathbb{R}^{d} \oplus \mathbb{R}^{d}\right)$ by

$$
\{f, g\}=\sum_{i=1}^{d}\left(\frac{\partial f}{\partial q_{i}} \frac{\partial g}{\partial p_{i}}-\frac{\partial f}{\partial p_{i}} \frac{\partial g}{\partial q_{i}}\right)
$$

for $f, g \in C^{\infty}\left(\mathbb{R}^{d} \oplus \mathbb{R}^{d}\right)$. Here $\left(q_{i}, p_{i}\right)$ are the coordinates on $\mathbb{R}^{d} \oplus \mathbb{R}^{d}$. The ordered pair $\left(C^{\infty}\left(\mathbb{R}^{d} \oplus \mathbb{R}^{d}\right),\{\cdot, \cdot\}\right)$ is an example of an involutive Poisson algebra in the sense that $C^{\infty}\left(\mathbb{R}^{d} \oplus \mathbb{R}^{d}\right)$ is a commutative ${ }^{*}$-algebra which is also a Lie algebra under the bracket $\{\cdot, \cdot\}$ satisfying in addition

$$
\{f, g h\}=\{f, g\} h+g\{f, h\}, \quad\{f, g\}^{*}=\left\{f^{*}, g^{*}\right\}
$$

for all $f, g, h \in C^{\infty}\left(\mathbb{R}^{d} \oplus \mathbb{R}^{d}\right)$. The bracket $\{\cdot, \cdot\}$ on $C^{\infty}\left(\mathbb{R}^{d} \oplus \mathbb{R}^{d}\right)$ is thus uniquely determined on the generating coordinate functions $q_{i}, p_{i} \in C^{\infty}\left(\mathbb{R}^{d} \oplus \mathbb{R}^{d}\right)$ on $\mathbb{R}^{d} \oplus \mathbb{R}^{d}$. Physically $q_{i}$ and $p_{i}$ represent the position and momentum observables respectively, say for the $i$ 'th particle in a 1-dimensional motion, and they clearly satisfy $\left\{q_{i}, p_{j}\right\}=\delta_{i j}$, whereas Heisenberg's commutation relation between the corresponding observable operators $P_{i}, Q_{i}$ in quantum mechanics is $\left[Q_{i}, P_{j}\right]=i \hbar \delta_{i j}$. With the discovery of the Weyl transform, it was realized that the replacement of $\{\cdot, \cdot\}$ by $[\cdot, \cdot]$ is correct for the functions $p_{i}$ and $q_{i}$, whereas for other functions it describes the non-commutative deformed product only up to the first order in $\hbar$.

A different approach to quantization was formulated by Rieffel, [44], who introduced the notion of a 'strict deformation quantization' within the setting of $\mathrm{C}^{*}$-algebras. This approach does not involve formal power series but rather a continuous field of $\mathrm{C}^{*}$-algebras, and is therefore considered an analytic deformation. 
Definition 6.1 Let $(A,\{\cdot, \cdot\})$ be an involutive Poisson algebra over $\mathbb{C}$ endowed with a $C^{*}$-norm. By a strict deformation quantization of $A$ in the direction of $\{\cdot, \cdot\}$, we mean an open interval $I$ of real numbers with 0 as center, together with, for each $\hbar \in I$, an associative product $\times_{\hbar}$, an involution $*_{\hbar}$, and a $C^{*}$-norm $\|\cdot\|_{\hbar}$ (for $\times_{\hbar}$ and $*_{\hbar}$ ) on $A$, which for $\hbar=0$ are the original product, involution and norm on $A$, such that:

- for every $a \in A$ the function $\hbar \mapsto\|a\|_{\hbar}$ is continuous,

- for every $a, b \in A$

$$
\left\|\left(a \times_{\hbar} b-b \times_{\hbar} a\right) / i \hbar-\{a, b\}\right\|_{\hbar} \rightarrow 0 \text { as } \hbar \rightarrow 0 .
$$

A. Bauval, [6], showed that $S U_{q}(2)$ is a strict deformation quantization (and also operator deformation quantization in the sense of A. J-L. Sheu, [51]) of the involutive Poisson algebra $(\operatorname{Pol}(S U(2)),\{\cdot, \cdot\})$ of regular functions on $S U(2)$ with bracket $\{\cdot, \cdot\}$ determined by:

$$
\{\alpha, \gamma\}=\frac{i}{2} \alpha \gamma,\left\{\alpha, \gamma^{*}\right\}=\frac{i}{2} \alpha \gamma^{*},\left\{\alpha, \alpha^{*}\right\}=-i \gamma \gamma^{*},\left\{\gamma, \gamma^{*}\right\}=0
$$

Here $\alpha$ and $\gamma$ are the generators of the unital $*$-algebra $\operatorname{Pol}(S U(2))$ of regular functions on the group $S U(2)$ introduced in Section 3 (part I). The involutive Poisson algebra $(\operatorname{Pol}(S U(2)),\{\cdot, \cdot\})$ has the additional feature of being an involutive Poisson Hopf algebra, [12], meaning that the co-multiplication $\Delta$ is a Poisson algebra homomorphism, i.e.

$$
\Delta(\{a, b\})=\{\Delta(a), \Delta(b)\}
$$

for all $a, b \in \operatorname{Pol}(S U(2))$. The Poisson bracket on the tensor product $\operatorname{Pol}(S U(2)) \odot \operatorname{Pol}(S U(2))$ is defined by

$$
\{a \otimes b, c \otimes d\}=a c \otimes\{b, d\}+\{a, c\} \otimes b d
$$

for all $a, b, c, d \in \operatorname{Pol}(S U(2))$. 
Let us transfer these notions to the dual side. For the moment we forget about the involution.

Let $G$ be a Lie group with Lie algebra $g$ and universal enveloping algebra $U(g)$. It is well known, [1], that $U(g)$ is a co-commutative Hopf algebra with co-multiplication $\hat{\Delta}: U(g) \rightarrow$ $U(g) \odot U(g)$ uniquely determined by

$$
\hat{\Delta}(X)=X \otimes 1+1 \otimes X
$$

for all $X \in g$. By definition $g$ is the vector space of left-invariant vector fields on the Lie group $G$, and $U(g)$ consists of the (higher order) left-invariant differential operators, [67], on $G$. Thus $U(g)$ can be thought of as consisting of the linear functionals on the algebra $C^{\infty}(G)$ of smooth (or analytic) functions on $G$ via the bilinear form $\langle\cdot, \cdot\rangle: U(g) \times C^{\infty}(G) \rightarrow \mathbb{C}$ defined by

$$
\langle X, f\rangle=X(f)(e)
$$

for all $X \in U(g)$ and $f \in C^{\infty}(G)$. Here $e \in G$ is the unit element of $G$.

Consider now a compact Lie group $G$ and the Hopf algebra $(\mathcal{A}, \Phi)$ of regular functions on $G$. The Hopf algebras $(U(g), \hat{\Delta})$ and $(\mathcal{A}, \Phi)$ form a dual pair for the restriction of the above form $\langle\cdot, \cdot\rangle$, and the co-multiplication $\hat{\Delta}$ reflects the derivation property of the generating left-invariant vector fields.

If $\mathcal{A}$ also has a bracket $\{\cdot, \cdot\}$ making $\mathcal{A}$ a Poisson Hopf algebra $(\mathcal{A}, \Phi,\{\cdot, \cdot\})$, we may dualize the bracket and obtain a linear map $\delta: U(g) \rightarrow U(g) \odot U(g)$ defined according to the rule

$$
\delta(X)(a \otimes b)=X(\{a, b\})
$$

for all $X \in U(g)$ and $a, b \in \mathcal{A}$. The triple $(U(g), \hat{\Delta}, \delta)$ constitutes what Drinfeld, [11], calls a co-Poisson Hopf algebra, and $\delta$ is compatible with the Hopf algebra structure in a sense dual to the compatibility of the bracket $\{\cdot, \cdot\}$ with the multiplication and co-multiplication on $(\mathcal{A}, \Phi)$. 
It is technically more convenient to quantize co-Poisson Hopf algebras. We are then deforming the co-commutative comultiplication $\hat{\Delta}$ instead of the multiplication on $U(g)$. Following Drinfeld we quantize formally, so let $\left(U_{\hbar}(g), \hat{\Delta}_{\hbar}\right)$ be a Hopf algebra over the ring $\mathbb{C}[[\hbar]]$. It is defined as an ordinary Hopf algebra with $\mathbb{C}$ replaced by $\mathbb{C}[[\hbar]]$. Strictly speaking, the algebra $U_{\hbar}(g)$ should be complete in the $\hbar$-adic topology, [22], which, for instance, allows for taking exponentials $\exp (\hbar X) \in U_{\hbar}(g)$ of elements $X \in U_{\hbar}(g)$. Naturally, tensor products should be topological ones and maps continuous etc. Drinfeld also requires $U_{\hbar}(g)$ to be topologically free as a $\mathbb{C}[[\hbar]]$-module. Denote by $\pi$ the canonical projection from $U_{\hbar}(g)$ to the quotient $U_{\hbar}(g) / \hbar U_{\hbar}(g)$, which is an ordinary Hopf algebra. Let $\sigma: U_{\hbar}(g) \otimes U_{\hbar}(g) \rightarrow U_{\hbar}(g) \otimes U_{\hbar}(g)$ denote the flip automorphism.

Definition 6.2 Let notation be as above. We say that $\left(U_{\hbar}(g), \hat{\Delta}_{\hbar}\right)$ is a quantization of the co-Poisson Hopf algebra $(U(g), \hat{\Delta}, \delta)$ if:

- $\pi\left(U_{\hbar}(g)\right)$ and $U(g)$ are isomorphic as Hopf algebras,

- $\delta(\pi(a))=(\pi \otimes \pi)\left(\frac{1}{i \hbar}\left(\hat{\Delta}_{\hbar}(a)-\sigma \hat{\Delta}_{\hbar}(a)\right)\right)$ for all $a \in U_{\hbar}(g)$.

The latter formula makes sense, because co-commutativity of $(U(g), \hat{\Delta})$ assures that $\hat{\Delta}_{\hbar}(a)-\sigma \hat{\Delta}_{\hbar}(a)$ belongs to the ideal $\hbar U_{\hbar}(g) \otimes U_{\hbar}(g)$ and, moreover, $\frac{1}{i \hbar}\left(\hat{\Delta}_{\hbar}(a)-\sigma \hat{\Delta}_{\hbar}(a)\right)$ is uniquely determined modulo $\hbar U_{\hbar}(g) \otimes U_{\hbar}(g)$.

To see how the actual quantization is constructed, it is instructive to restrict to the simplest non-trivial example $\operatorname{sl}(2)$, which is fundamental in the theory of complex semi-simple Lie algebras. This is the Lie algebra of the special linear group $S L(2)$ and consists of complex $2 \times 2$-matrices of zero trace. It is a 3 -dimensional complex simple Lie algebra with linear basis $H, E_{+}, E_{-}$given by

$$
H=\left(\begin{array}{cc}
1 & 0 \\
0 & -1
\end{array}\right), \quad E_{+}=\left(\begin{array}{ll}
0 & 1 \\
0 & 0
\end{array}\right), \quad E_{-}=\left(\begin{array}{ll}
0 & 0 \\
1 & 0
\end{array}\right)
$$


The Lie bracket $[\cdot, \cdot]$ on $\operatorname{sl}(2)$ is thus determined by

$$
\left[E_{+}, E_{-}\right]=H, \quad\left[H, E_{ \pm}\right]= \pm 2 E_{ \pm} .
$$

In fact, $s l(2)$ is the universal Lie algebra with this bracket. The formulas

$$
\delta(H)=0, \quad \delta\left(E_{ \pm}\right)=-\frac{i}{2}\left(E_{ \pm} \otimes H-H \otimes E_{ \pm}\right)
$$

determine a linear map $\delta: U(s l(2)) \rightarrow U(s l(2)) \odot U(s l(2))$ making $(U(\operatorname{sl}(2)), \hat{\Delta}, \delta)$ a co-Poisson Hopf algebra, [18]. As we shall see later $\delta$ is closely connected to the Poisson bracket on $S U(2)$ which gave rise to the strict quantization deformation $S U_{q}(2)$ mentioned earlier. Now in order to quantize $(U(\operatorname{sl}(2)), \hat{\Delta}, \delta)$, we need to construct (in some sense universal) unital algebra $U_{\hbar}(\operatorname{sl}(2))$ with a map $\hat{\Delta}_{\hbar}$ on $U_{\hbar}(\operatorname{sl}(2))$ satisfying certain conditions.

Before we proceed let us make some remarks concerning topologically freeness of $U_{\hbar}(s l(2))$. This condition means (see p.395 in [22]) that as a left $\mathbb{C}[[\hbar]]$-module $U_{\hbar}(\operatorname{sl}(2))$ is isomorphic to the left $\mathbb{C}[[\hbar]]$-module $\left(U_{\hbar}(s l(2)) / \hbar U_{\hbar}(s l(2))\right)[[\hbar]]$ of formal power series in the variable $\hbar$ with coefficients in the quotient algebra $U_{\hbar}(s l(2)) / \hbar U_{\hbar}(s l(2))$. By hypothesis, we have $U_{\hbar}(\operatorname{sl}(2)) / \hbar U_{\hbar}(\operatorname{sl}(2))=U(\operatorname{sl}(2))$, so

$$
U_{\hbar}(\operatorname{sl}(2))=U(\operatorname{sl}(2))[[\hbar]]
$$

as a left $\mathbb{C}[[\hbar]]$-module. Hence the $n$-fold tensor power of $\left.U_{\hbar}(\operatorname{sl}(2))\right)$ is given by $U_{\hbar}(\operatorname{sl}(2))^{\otimes n}=\left(U(\operatorname{sl}(2))^{\odot n}\right)[[\hbar]]$.

Without loss of generality, we may therefore impose the ansatz

$$
\hat{\Delta}_{\hbar}=\sum_{n=0}^{\infty} \frac{\hbar^{n}}{n !} \hat{\Delta}_{n}
$$

where $\hat{\Delta}_{n}: U(\operatorname{sl}(2)) \rightarrow U(s l(2)) \odot U(s l(2))$ are linear maps. By this we mean of course that for a typical element

$$
a=\sum_{n=0}^{\infty} a_{n} \hbar^{n} \in U(\operatorname{sl}(2))[[\hbar]]=U_{\hbar}(\operatorname{sl}(2)),
$$


with coefficients $a_{m} \in U(\operatorname{sl}(2))$, we have

$\Delta_{\hbar}(a)=\sum_{n=0}^{\infty} \Delta_{n}\left(a_{n}\right) \hbar^{n} \in(U(s l(2)) \odot U(s l(2)))[[\hbar]]=U_{\hbar}(s l(2)) \otimes U_{\hbar}(s l(2))$.

Let $\sigma$ denote the flip on $U(\operatorname{sl}(2)) \odot U(s l(2))$. The two listed requirements in the definition above give the constraints $\hat{\Delta}_{0}=\hat{\Delta}$ and $\delta=-i\left(\hat{\Delta}_{1}-\sigma \hat{\Delta}_{1}\right)$, while the maps $\hat{\Delta}_{n}$ remain undetermined for $n \geq 2$. However, the co-associative requirement of $\hat{\Delta}_{\hbar}$ gives the recursion relations

$$
\sum_{j=0}^{n}\left(\begin{array}{c}
n \\
j
\end{array}\right)\left(\hat{\Delta}_{j} \otimes \iota-\iota \otimes \hat{\Delta}_{j}\right) \hat{\Delta}_{n-j}=0
$$

for $n \geq 2$. To see this notice that

$$
\left(\hat{\Delta}_{\hbar} \otimes i d\right) \hat{\Delta}_{\hbar}=\sum_{n=0}^{\infty} \hbar^{n} \sum_{j=0}^{n} \frac{1}{j !(n-j) !}\left(\hat{\Delta}_{j} \otimes \iota\right) \hat{\Delta}_{n-j} .
$$

An obvious solution to the constraint on $\hat{\Delta}_{1}$ is $\hat{\Delta}_{1}=\frac{i}{2} \delta$, and this is the only known solution apart from the trivial possibility of adding terms that are $\sigma$-invariant. Fixing this solution, inspection and an application of the formula

$$
\hat{\Delta}_{0}\left(H^{n}\right)=\sum_{j=0}^{n}\left(\begin{array}{l}
n \\
j
\end{array}\right) H^{n-j} \otimes H^{j}
$$

for any natural number $n$, reveal that the recursion relations are all solved simultaneously by taking

$$
\hat{\Delta}_{n}(H)=0, \quad \hat{\Delta}_{n}\left(E_{ \pm}\right)=2^{-2 n}\left(E_{ \pm} \otimes H^{n}+(-1)^{n} H^{n} \otimes E_{ \pm}\right)
$$

for all $n \geq 1$. Inserting these multi solutions into the ansatz for $\hat{\Delta}_{\hbar}$, we get the co-product rules:

$$
\hat{\Delta}_{\hbar}(H)=H \otimes I+I \otimes H,
$$




$$
\hat{\Delta}_{\hbar}\left(E_{ \pm}\right)=E_{ \pm} \otimes e^{\hbar H / 4}+e^{-\hbar H / 4} \otimes E_{ \pm}
$$

which again uniquely determine the co-unit and the co-inverse. Finally, the algebra structure or the commutation relations for the generators of $U_{\hbar}(s l(2))$ can be found by imposing the homomorphism property of $\hat{\Delta}_{\hbar}$, namely,

$\hat{\Delta}_{\hbar}\left(\left[H, E_{ \pm}\right]\right)=\left[\hat{\Delta}_{\hbar}(H), \hat{\Delta}_{\hbar}\left(E_{ \pm}\right)\right]=\left[H, E_{ \pm}\right] \otimes e^{\hbar H / 4}+e^{-\hbar H / 4} \otimes\left[H, E_{ \pm}\right]$,

which is satisfied by taking $\left[H, E_{ \pm}\right]=c( \pm) E_{ \pm}$for elements $c( \pm) \in$ $\mathbb{C}[[\hbar]]$. When exponentiated these relations read $e^{u \hbar H} E_{ \pm}=$ $e^{c( \pm) u \hbar} E_{ \pm} e^{u \hbar H}$ for any $u \in \mathbb{C}$. The expression for $\hat{\Delta}_{\hbar}\left(\left[E_{+}, E_{-}\right]\right)=$ $\left[\hat{\Delta}\left(E_{+}\right), \hat{\Delta}\left(E_{-}\right)\right]$therefore reduces to

$$
\hat{\Delta}_{\hbar}\left(\left[E_{+}, E_{-}\right]\right)=\left[E_{+}, E_{-}\right] \otimes e^{\hbar H / 2}+e^{-\hbar H / 2} \otimes\left[E_{+}, E_{-}\right],
$$

when $c=c(+)=-c(-)$. This equation holds if we take $\left[E_{+}, E_{-}\right]=v\left(e^{\hbar H / 2}-e^{-\hbar H / 2}\right)$ for any $v \in \mathbb{C}[[\hbar]]$.

Only the leading terms in the elements $c, v \in \mathbb{C}[[\hbar]]$ can be fixed, namely by insisting on the correct behavior in the classical limit. The specific choices $c=2$ and $v=\frac{1}{2}\left(\sinh \left(\frac{\hbar}{2}\right)\right)^{-1}$ (although not really a member of $\mathbb{C}[[\hbar]]$, it gives a well-defined expression for the commutator $\left[E_{+}, E_{-}\right]$written below) give the commutation relations:

$$
\left[H, E_{ \pm}\right]= \pm 2 E_{ \pm}, \quad\left[E_{+}, E_{-}\right]=\frac{1}{e^{\hbar / 2}-e^{-\hbar / 2}}\left(e^{\hbar H / 2}-e^{-\hbar H / 2}\right)
$$

valid in $U_{\hbar}(s l(2))$, while any other choice consistent with the classical limit leads to an isomorphic algebra. Of course, the algebra $U_{\hbar}(s l(2))$ is defined to be the algebra topologically generated by $H, E_{ \pm}$satisfying these commutation relations. It is easy to check that the formulas for $\hat{\Delta}_{\hbar}(H)$ and $\hat{\Delta}_{\hbar}\left(E_{ \pm}\right)$stated above define a co-multiplication $\hat{\Delta}_{\hbar}$ on the algebra $U_{\hbar}(s l(2))$ thus defined.

M. Jimbo, [20], observed that one can derive an ordinary Hopf algebra $\left(U_{q}(s l(2)), \hat{\Delta}\right)$ from the pair $\left(U_{\hbar}(s l(2)), \hat{\Delta}_{\hbar}\right)$. Instead 
of working with the generator $H$ for $U_{\hbar}(s l(2))$, which forces one to consider infinite power series (in order to include for instance the elements $e^{\hbar H / 4}$ in $\left.U_{\hbar}(s l(2))\right)$, he regarded $\hbar \in U_{\hbar}(s l(2))$ as a fixed complex number and considered the complex universal algebra $U_{q}(s l(2))$ with the exponentials $e^{ \pm \hbar H / 4}$ and $E_{ \pm}$as generators. More precisely, let

$$
q=e^{-\hbar / 2}, \quad k=e^{-\hbar H / 4}, \quad k^{-1}=e^{\hbar H / 4}, \quad e=E_{+}, \quad f=E_{-} .
$$

The relations between these generators are dictated by those for $H, E_{ \pm}$in $U_{\hbar}(s l(2))$, and are therefore

$k k^{-1}=k^{-1} k=I, \quad k e=q e k, k f=q^{-1} f k, \quad[e, f]=\frac{1}{q-q^{-1}}\left(k^{2}-k^{-2}\right)$,

whereas the co-multiplication $\hat{\Delta}: U_{q}(\operatorname{sl}(2)) \rightarrow U_{q}(\operatorname{sl}(2)) \odot$ $U_{q}(\operatorname{sl}(2))$ is determined by

$\hat{\Delta}(k)=k \otimes k, \quad \hat{\Delta}(e)=e \otimes k^{-1}+k \otimes e, \quad \hat{\Delta}(f)=f \otimes k^{-1}+k \otimes f$.

Let us return to the question of defining an involution on these objects. It is well known that the special unitary group $S U(2)$ is the maximal compact subgroup of $S L(2)$. It is a 3dimensional real Lie group with real Lie algebra $s u(2)$ consisting of complex skew-symmetric $2 \times 2$-matrices of zero trace. In fact, $s u(2)$ is the compact real form of $s l(2)$, so its complexification is $\operatorname{sl}(2)$ and furthermore the restriction of the Cartan-Killing form on $s l(2)$ to $s u(2)$ is strictly non-negative definite. Another way of saying this is to introduce the standard anti-linear Cartan involution $\omega$ on $\operatorname{sl}(2)$ defined by

$$
\omega(H)=-H, \quad \omega\left(E_{+}\right)=-E_{-} .
$$

Then $s u(2)$ is just the set of fixed points under this involution. The famous Pauli-matrices

$$
e_{1}=\left(\begin{array}{cc}
i & 0 \\
0 & -i
\end{array}\right), \quad e_{2}=\left(\begin{array}{cc}
0 & 1 \\
-1 & 0
\end{array}\right), \quad e_{3}=\left(\begin{array}{cc}
0 & i \\
i & 0
\end{array}\right)
$$


constitute a basis for the real vector space $s u(2)$. So we may regard $s u(2)$ as the complex Lie algebra $s l(2)$ together with the involution $\omega$. By universality we may extend $\omega$ uniquely to an anti-linear involution $\omega$ on $U(g)$ and thus the real Hopf algebra $(U(s u(2)), \hat{\Delta})$ is uniquely determined by the triple $(U(s l(2)), \hat{\Delta}, \omega)$.

What about the quantum case? When $q$ is real, there is an anti-linear involution $\omega_{q}$ on $U_{q}(\operatorname{sl}(2))$ given by

$$
\omega_{q}(k)=k^{-1}, \quad \omega_{q}(e)=-q^{-1} f .
$$

It can be used to introduce the more conventional *-operation on $\left(U_{q}(s l(2)), \hat{\Delta}\right)$ given by $a^{*}=\omega_{q}\left(S^{-1}(a)\right)$ for all $a \in U_{q}(\operatorname{sl}(2))$. Here $S$ is the antipode on $U_{q}(\operatorname{sl}(2))$. Henceforth we regard $\left(U_{q}(s u(2)), \hat{\Delta}\right)$ as the triple $\left(U_{q}(s l(2)), \hat{\Delta},{ }^{*}\right)$, because we may recover the triple $(U(\operatorname{sl}(2), \hat{\Delta}, \omega)$ and thus $s u(2)$ in the classical limit $q \rightarrow 1$. The ${ }^{*}$-operation on $U_{q}(\operatorname{sl}(2))$ is determined by the formulas $k^{*}=k$ and $e^{*}=f$.

Now there are two known ways to produce the unital Hopf *algebra $(\mathcal{A}, \Phi)$ of regular functions on the quantum group $S U_{q}(2)$ from $\left(U_{q}(s l(2)), \hat{\Delta},{ }^{*}\right)$. We begin by explaining the first method. It was suggested by Drinfeld in [11] and developed extensively by Y. Soibelman, L. L. Vaksman and S. Levendorski, [52], [32],[59], (also used by M. Rosso, [48], to clarify the relationship between the Drinfeld and Woronowicz approaches to quantum $S U(2))$, namely to let $\mathcal{A}$, as a vector space, be the linear span of the matrix elements of all finite-dimensional $*$-representations of $\left(U_{q}(\operatorname{sl}(2)), \hat{\Delta},{ }^{*}\right)$.

Clearly the matrix elements of these representations are linear functionals on the Hopf ${ }^{*}$-algebra $\left(U_{q}(s l(2)), \hat{\Delta},{ }^{*}\right)$, so the Hopf *-algebra structure on $\mathcal{A}$ is the one dual to the Hopf *-algebra $\left(U_{q}(\operatorname{sl}(2)), \hat{\Delta},{ }^{*}\right)$. It is a theorem, see [59], that the Hopf *-algebra thus obtained is isomorphic to the $\operatorname{Hopf}{ }^{*}$-algebra $(\mathcal{A}, \Phi)$ of regular functions defined in Section 3 (part I). The important ingredient here is that there are enough finite-dimensional *-representations on $\left(U_{q}(s l(2)), \hat{\Delta},{ }^{*}\right)$ to separate the elements of $U_{q}(\operatorname{sl}(2))$, so we 
have a non-degenerate pairing of Hopf *-algebras. Later we will list these representations. Now we settle for emphasizing that they form a strict tensor $\mathrm{C}^{*}$-category $\mathcal{T}$ with conjugates. Of course, we are here just obtaining (in the sense of the generalized Tannaka-Krein theorem of Woronowicz) the pair $(\mathcal{A}, \Phi)$ as the compact quantum group whose strict tensor $\mathrm{C}^{*}$-category of finite-dimensional unitary co-representations is equivalent to $\mathcal{T}$. The formulas

$$
u(k)=\left(\begin{array}{cc}
q^{1 / 2} & 0 \\
0 & q^{-1 / 2}
\end{array}\right), u(e)=\left(\begin{array}{ll}
0 & 1 \\
0 & 0
\end{array}\right), u(f)=\left(\begin{array}{ll}
0 & 0 \\
1 & 0
\end{array}\right)
$$

define a 2-dimensional ${ }^{*}$-representation of $\left(U_{q}(\operatorname{sl}(2)), \hat{\Delta},{ }^{*}\right)$. Every finite-dimensional ${ }^{*}$-representation of $\left(U_{q}(s l(2)), \hat{\Delta},{ }^{*}\right)$ is contained (as a subrepresentation) in tensor products of $u$ and its conjugate $\bar{u}$. Clearly $u$ also plays the role of the fundamental unitary co-representation in the definition of the compact matrix pseudo group $(\mathcal{A}, u)$ from Section 3 (part I). Let $u_{i j}$ be the matrix elements of $u$. The ${ }^{*}$-operation on $\mathcal{A}$ is uniquely determined by $u_{11}^{*}=u_{22}$ and $u_{12}^{*}=-q u_{21}$, and finally we get the familiar relations from Section 3 (part I) for the generators $\alpha=u_{11}$ and $\gamma=u_{21}$ of the unital *-algebra $\mathcal{A}$. We will explain the second method for constructing $(\mathcal{A}, \Phi)$ from $\left(U_{q}(s l(2)), \hat{\Delta},{ }^{*}\right)$ later when we have defined $R$-matrices.

Consider the classical limit $q \rightarrow 1$. The fundamental representation $u$ of the Hopf *-algebra $\left(U(\operatorname{sl}(2)), \hat{\Delta},{ }^{*}\right)$ is ${ }^{*}$-preserving, where now $H^{*}=H$ and $E_{+}^{*}=E_{-}$. Trivial calculation shows that the involutive co-Poisson Hopf algebra $\left(U(\operatorname{sl}(2)), \hat{\Delta},{ }^{*}, \delta\right)$ that we formally quantized is dual (in the sense described earlier) to the involutive Poisson Hopf algebra $(\operatorname{Pol}(S U(2)), \Phi,\{\cdot, \cdot\})$ that Bauval considered, and which had $S U_{q}(2)$ as a strict deformation quantization.

It turns out that this involutive Poisson Hopf algebra $(\operatorname{Pol}(S U(2)), \Phi,\{\cdot, \cdot\})$ can be derived from the element

$$
r=\frac{1}{4} H \otimes H+E_{+} \otimes E_{-} \in \operatorname{sl}(2) \odot \operatorname{sl}(2) \subset U(\operatorname{sl}(2)) \odot U(\operatorname{sl}(2)) .
$$


By the duality between the Hopf *-algebras $\left(U_{q}(\operatorname{sl}(2)), \hat{\Delta},{ }^{*}\right)$ and $(\operatorname{Pol}(S U(2)), \Phi)$ mentioned above, we may and do regard the elements of $\operatorname{sl}(2)$ as linear functionals of the algebra $\operatorname{Pol}(S U(2))$. For $X \in \operatorname{sl}(2)$ define therefore the left-invariant vector field $\hat{X}$ and the right-invariant vector field $\tilde{X}$ as endomorphisms on $\operatorname{Pol}(S U(2))$ given by

$$
\hat{X}=(\iota \otimes X) \Phi, \quad \tilde{X}=(X \otimes \iota) \Phi .
$$

Let $\sigma$ denote the flip on $U(\operatorname{sl}(2)) \odot U(\operatorname{sl}(2))$. Set

$$
r_{a}=-\frac{i}{2}(r-\sigma r)=-\frac{i}{2}\left(E_{+} \otimes E_{-}-E_{-} \otimes E_{+}\right)
$$

and

$\overline{r_{a}}=\hat{r_{a}}-\tilde{r_{a}}=-\frac{i}{2}\left(\hat{E_{+}} \otimes \hat{E_{-}}-\hat{E_{-}} \otimes \hat{E_{+}}-\tilde{E_{+}} \otimes \tilde{E_{-}}+\tilde{E_{-}} \otimes \tilde{E_{+}}\right)$.

One may check that the Poisson bracket $\{\cdot, \cdot\}$ on $\operatorname{Pol}(S U(2))$ is given by

$$
\{a, b\}=\overline{r_{a}}(a \otimes b)
$$

for all $a, b \in \operatorname{Pol}(S U(2))$.

The fact that $\{\cdot, \cdot\}$ is a Poisson bracket, is reflected in the equation

$$
\left[r_{12}, r_{13}\right]+\left[r_{12}, r_{23}\right]+\left[r_{13}, r_{23}\right]=0 \text {, }
$$

satisfied by $r$, known as the classical Yang Baxter equation (CYBE), [11]. As usual $r_{12}=r \otimes I, r_{23}=I \otimes r$ and $r_{13}=(\iota \otimes$ $\sigma)\left(r_{12}\right)$ considered as elements of $U(\operatorname{sl}(2)) \odot U(\operatorname{sl}(2)) \odot U(\operatorname{sl}(2))$. Clearly the CYBE is satisfied for the anti-symmetrized version $r_{a}$ as well.

Drinfeld and A. A. Belavin, [11], have classified all solutions of the CYBE that yield Poisson Hopf algebras on any given simply connected simple (and semisimple) complex Lie group. Drinfeld has further shown that all such Poisson structures arise in this way. The involutive Poisson Hopf algebras on their maximal compact subgroups are therefore also classified, [32]. There are three 
different classes of Poisson brackets on each such compact group, where one of them is called the standard one. The $\delta$ which we considered for the $s l(2)$-case with associated Lie group $S L(2)$ and maximal compact subgroup $S U(2)$, comes from the standard class solution.

All these Poisson Hopf algebras admit formal quantizations which are essentially unique, [32]. The relations for their generators are similar to the ones for $U_{\hbar}(s l(2))$ and involve the Cartan matrix for the associated Lie algebra, which occurs trivially as the number 2 in the relations for $U_{\hbar}(\operatorname{sl}(2))$. In fact, the formal quantizations of Drinfeld also include quantizations of certain infinite-dimensional Lie algebras, namely the Kac-Moody algebras, [11],[18], where the determinant condition on the Cartan matrix involved is dropped. As in the classical theory, for an arbitrary complex simple Lie algebra, one gets the additional Serre relations, which vanish identically for the case $s l(2)$ also in the classical limit. Most properties from the classical theory survive quantization, for instance, the quantizations mentioned above have PoincareBirkhoff-Witt-type-bases and Weyl groups. Also their representation theory is very much the same as for their classical origins; with Casimir elements generating their centres and which separate their finite-dimensional irreducible representations, and a classification of their finite-dimensional representations in terms of highest weights. For the Jimbo algebras, however, deviations from the classical representation theory occur when the deformation parameter $q$ is a root of unity, [22], and for this reason (among others) these cases have received special interest. However, they do not correspond to $\mathrm{C}^{*}$-algebraic quantum groups, so we will continue to restrict to the case when $q$ is real.

The solution $r \in U(\operatorname{sl}(2)) \odot U(\operatorname{sl}(2))$ of the CYBE has a quantum counterpart $R \in U_{\hbar}(s l(2)) \otimes U_{\hbar}(s l(2))$, which is a formal quantization of $r$ in the sense that

$$
r=\frac{1}{\hbar}(R-I \otimes I) \bmod \hbar
$$

The element $R$ satisfies the quantum Yang Baxter equation 
(QYBE)

$$
R_{12} R_{13} R_{23}=R_{23} R_{13} R_{12},
$$

where $R_{12}=R \otimes I \in U_{\hbar}(s l(2)) \otimes U_{\hbar}(s l(2)) \otimes U_{\hbar}(s l(2))$, and so on.

In fact, there exists an invertible element $R \in U_{\hbar}(\operatorname{sl}(2)) \otimes$ $U_{\hbar}(\operatorname{sl}(2))$ which satisfies the equations

$$
\begin{gathered}
\sigma \hat{\Delta}_{\hbar}=R \hat{\Delta}_{\hbar} R^{-1} \\
\left(\hat{\Delta}_{\hbar} \otimes i d\right)(R)=R_{13} R_{23}, \quad\left(i d \otimes \hat{\Delta}_{\hbar}\right)(R)=R_{13} R_{12}
\end{gathered}
$$

and is a formal quantization of $r$. The latter two equations above and the co-associativity of $\hat{\Delta}_{\hbar}$ immediately imply that $R$ satisfies the QYBE. To construct $R$ one may propose an ansatz for $R$ similar to that of $\hat{\Delta}_{\hbar}$ and in principle proceed as before but now requiring $R$ to satisfy the QYBE. Drinfeld, [11], uses the notion of quasi-triangular Hopf algebra $(A, \Delta, R)$ for a Hopf algebra supplied with an element $R \in A \otimes A$ fulfilling the algebraic properties described above. The prefixes triangular and co-boundary are used for modifications of such Hopf algebras.

However, to construct $R \in U_{\hbar}(s l(2)) \otimes U_{\hbar}(s l(2))$, it is more convenient to apply the recipe given by Drinfeld. This method, called a quantum-double construction, involves calculations that are manageable. It is called a double construction because it involves two Hopf algebras obtained by restricting $\hat{\Delta}_{\hbar}$ to the subalgebras $U_{\hbar}\left(b_{ \pm}\right)$of $U_{\hbar}(s l(2))$ given by

$$
U_{\hbar}\left(b_{ \pm}\right)=\operatorname{span}\left\{H^{m} E_{ \pm}^{n} \mid m, n=0,1,2, \ldots\right\} .
$$

They correspond to the Borel subalgebras $b_{ \pm}$of the Lie algebra $\operatorname{sl}(2)$ which are given by $b_{ \pm}=\operatorname{span}\left\{H, E_{ \pm}\right\}$. Using the fact that the linear generators for the vector spaces $U_{\hbar}\left(b_{ \pm}\right)$are linear independent, one may prove that $U_{\hbar}\left(b_{+}\right)$and $U_{\hbar}\left(b_{-}\right)$are dual to each other as vector spaces, [18]. Pick dual bases $\left\{e_{i}\right\} \subset U_{\hbar}\left(b_{+}\right)$and $\left\{e^{i}\right\} \subset U_{\hbar}\left(b_{-}\right)$and form the element

$$
R=\sum_{i=0}^{\infty} e_{i} \otimes e^{i} \in U_{\hbar}(s l(2)) \otimes U_{\hbar}(s l(2)) .
$$


This element satisfies the properties specified above for $R$. The following concrete formula may be deduced, [18];

$$
R=e^{\frac{\hbar}{4} H \otimes H} \sum_{n=0}^{\infty} \frac{\left(e^{\frac{\hbar}{2}}-e^{-\frac{\hbar}{2}}\right)^{n}}{[n] !} e^{-\frac{\hbar}{4} n(n+1)} e^{\frac{\hbar n H}{4}} E_{+}^{n} \otimes e^{-\frac{\hbar n H}{4}} E_{-}^{n},
$$

where $[n]$ ! is the factorial $[n] !=\prod_{m=1}^{n}[m]$ of the celebrated $q$-numbers $[m]$ defined as $[m]=\left(e^{\frac{m \hbar}{2}}-e^{-\frac{m \hbar}{2}}\right) /\left(e^{\frac{\hbar}{2}}-e^{-\frac{\hbar}{2}}\right)$. Such $R$-elements with similar explicit formulas, are found for any of the above-mentioned quantizations $\left(U_{\hbar}(g), \hat{\Delta}_{\hbar}\right)$, [32], using the quantum double construction associated to the Borel subalgebras of the complex simple Lie algebra $g$.

The quantum double $D(A)$ is a Hopf algebra that can be formed from any finite-dimensional Hopf algebra $(A, \Delta)$, namely as the bi-crossed product, [22], of $(A, \Delta)$ and its opposite dual Hopf algebra which are matched by the adjoint representations. If $(A, \Delta)$ is co-commutative, this bi-crossed product is an ordinary crossed product. In the general case an element $R \in D(A) \otimes$ $D(A)$ may be formed which satisfies the QYBE. The notions of crossed products and quantum doubles have also been defined in the $C^{*}$-algebra context, [42].

The element $R \in U_{\hbar}(\operatorname{sl}(2)) \otimes U_{\hbar}(\operatorname{sl}(2))$ is called the universal $R$-matrix because it generates an abundance of $R$-matrices, which by definition are matrices solving the QYBE. In fact, Drinfeld constructed the universal $R$-matrix as a remedy to solve the difficult problem of finding all $R$-matrices.

Suppose $(A, \Delta, R)$ is a quasi-triangular Hopf algebra, and let $\pi_{i}:=A \rightarrow B\left(H_{i}\right), i \in\{1,2\}$, be finite-dimensional representations of $(A, \Delta, R)$. Then clearly $\mathcal{R}=\left(\pi_{1} \otimes \pi_{1}\right)(R)$ is an $R$-matrix. Hence every finite-dimensional representation of $(A, \Delta, R)$ produces an $R$-matrix.

Let $\Sigma$ denote the flip on $H_{1} \odot H_{2}$. Then $\Sigma \in B\left(H_{1} \odot\right.$ $\left.H_{2}, H_{2} \odot H_{1}\right)$. Observe that $\Sigma \mathcal{R} \Sigma=\sigma \mathcal{R}$, where $\sigma$ denotes the flip on $B\left(H_{1}\right) \odot B\left(H_{1}\right)=B\left(H_{1} \odot H_{1}\right)$. Write $\hat{\mathcal{R}}\left(\pi_{1}, \pi_{2}\right)$ for the element 
$\Sigma\left(\pi_{1} \otimes \pi_{2}\right)(R)$, where we have used the identification $B\left(H_{1}\right) \odot$ $B\left(H_{2}\right)=B\left(H_{1} \odot H_{2}\right)$. It is straightforward to check that $\hat{\mathcal{R}}=$ $\hat{\mathcal{R}}\left(\pi_{1}, \pi_{1}\right)=\Sigma \mathcal{R} \in B\left(H_{1}\right) \odot B\left(H_{1}\right)$ satisfies the following variant of the QYBE;

$$
\hat{\mathcal{R}}_{12} \hat{\mathcal{R}}_{23} \hat{\mathcal{R}}_{12}=\hat{\mathcal{R}}_{23} \hat{\mathcal{R}}_{12} \hat{\mathcal{R}}_{23},
$$

and for this reason $\hat{\mathcal{R}}$ is (with the risk of causing confusion) also commonly referred to as an $R$-matrix, [35].

Recall that the category of finite-dimensional representations of $(A, \Delta, R)$ is a monoidal category with product $\times$ given by

$$
\pi_{1} \times \pi_{2}=\left(\pi_{1} \otimes \pi_{2}\right) \Delta,
$$

for any finite-dimensional representations $\pi_{i}$ of $(A, \Delta, R)$. Thus the quasi-co-commutativity governed by $R$ ensures that $\left(\pi_{1}, \pi_{2}\right) \rightarrow$ $\hat{\mathcal{R}}\left(\pi_{1}, \pi_{2}\right)$ is a braiding for the monoidal category of finitedimensional representations of $(A, \Delta, R)$, [22].

Where does the braiding come in? First consider the trivial case when $R=I \otimes I$, so $\left(\hat{\mathcal{R}}_{12}\right)^{2}=I \otimes I \otimes I$. Form the $n$-fold tensor product $B^{\odot n}$ of $B=B\left(H_{1}\right)$ with itself, and denote by $\rho_{n}(i j) \in B^{\odot n}$ the element obtained from $\hat{\mathcal{R}}$ in the obvious way. Consider an element $s$ in the symmetric group $S_{n}$. Decompose $s$ into a product of transpositions $(i j)$ and form the product $\rho_{n}(s)$ of the corresponding elements $\rho_{n}(i j) \in B^{\odot n}$. The element $\rho_{n}(s)$ is independent of the decomposition of $s$ into transpositions, because the Coxeter relations

$$
\left(\hat{\mathcal{R}}_{12}\right)^{2}=I \otimes I \otimes I, \quad \hat{\mathcal{R}}_{12} \hat{\mathcal{R}}_{23} \hat{\mathcal{R}}_{12}=\hat{\mathcal{R}}_{23} \hat{\mathcal{R}}_{12} \hat{\mathcal{R}}_{23}
$$

form a presentation of the symmetric group $S_{3}$. Therefore we get a representation

$$
\rho_{n}: S_{n} \rightarrow B^{\odot n}=B\left(H^{\odot n}\right)
$$

of $S_{n}$ on $H^{\odot n}$. So the flip $\Sigma$ produces a representation of every $S_{n}$. 
In the more general case when $\left(\hat{\mathcal{R}}_{12}\right)^{2} \neq I \otimes I \otimes I$, we don't get a representation of the symmetric group $S_{n}$ on $B\left(H^{\odot n}\right)$, but rather of the infinite Artin braid group $B d_{n}$, i.e. the group formed by composing braids of width $n$. For the precise definitions see [18] and [22].

Let us embark on the second way of obtaining the Hopf *algebra $(\mathcal{A}, \Phi)$ from say the Hopf *-algebra $\left(U_{\hbar}(s l(2)), \hat{\Delta}_{\hbar}\right)$. As we have seen $U_{\hbar}(s l(2))$ contains a universal $R$-matrix for which we have written down a concrete formula. We have also seen that a given finite-dimensional representation $\pi_{1}$ of a quasi-triangular Hopf algebra produces an $R$-matrix $\mathcal{R}$. Similarly, a matrix representation $\pi_{1}$ (with coefficients in the commutative ring $\mathbb{C}[[\hbar]]$ ) of the algebra $U_{\hbar}(s l(2))$ yields an $R$-matrix $\mathcal{R}$. Now the formulas

$$
\pi_{1}(H)=\left(\begin{array}{cc}
1 & 0 \\
0 & -1
\end{array}\right), \pi_{1}\left(E_{+}\right)=\left(\begin{array}{cc}
0 & 1 \\
0 & 0
\end{array}\right), \pi_{1}\left(E_{-}\right)=\left(\begin{array}{cc}
0 & 0 \\
1 & 0
\end{array}\right)
$$

define a 2-dimensional (as a free $\mathbb{C}[[\hbar]]$-module) representation $\pi_{1}$ of $U_{\hbar}(s l(2))$. Note that the deformation parameter $\hbar$ does not enter in the formulas above, so the elements $\pi_{1}\left(E_{ \pm}\right), \pi_{1}(H)$ form a Lie algebra isomorphic to $s l(2)$ and the quantization is somehow hidden! Recalling the identification of the generators $E_{ \pm}, H$ for the algebra $U_{\hbar}(s l(2))$ and the generators $e, f, k^{ \pm}$for the Jimbo algebra $U_{q}(s l(2))$, we see that $\pi_{1}$ corresponds to the fundamental ${ }^{*}$-representation $u$ of the Hopf ${ }^{*}$-algebra $\left(U_{q}(s l(2)), \hat{\Delta}_{q},{ }^{*}\right)$.

We will need a concrete formula for $\mathcal{R}=\left(\pi_{1} \otimes \pi_{1}\right) R \in$ $M_{2}(\mathbb{C}[[\hbar]]) \otimes M_{2}(\mathbb{C}[[\hbar]])$. Since $\pi_{1}\left(E_{ \pm}\right)$both have square zero, we get

$$
\mathcal{R}=\left(\pi_{1} \otimes \pi_{1}\right)\left[e^{\frac{\hbar}{4} H \otimes H}\left(I \otimes I+\left(1-e^{-\hbar}\right) e^{\frac{\hbar}{4} H} E_{+} \otimes e^{-\frac{\hbar}{4} H} E_{-}\right)\right] .
$$

The formula

$$
e^{\frac{\hbar}{4} H \otimes H}=\sum_{n=0}^{\infty} \frac{\left(\frac{\hbar}{4} H \otimes H\right)^{n}}{n !}
$$


gives $\left(\pi_{1} \otimes \pi_{1}\right) e^{\frac{\hbar}{4} H \otimes H}$

$$
\begin{aligned}
& =\sum_{n \text { even }} \frac{\left(\frac{\hbar}{4}\right)^{n}}{n !}\left(\begin{array}{ll}
1 & 0 \\
0 & 1
\end{array}\right) \otimes\left(\begin{array}{ll}
1 & 0 \\
0 & 1
\end{array}\right)+\sum_{n \text { odd }} \frac{\left(\frac{\hbar}{4}\right)^{n}}{n !}\left(\begin{array}{cc}
1 & 0 \\
0 & -1
\end{array}\right) \otimes\left(\begin{array}{cc}
1 & 0 \\
0 & -1
\end{array}\right) \\
& =\cosh \frac{\hbar}{4}\left(\begin{array}{ll}
1 & 0 \\
0 & 1
\end{array}\right) \otimes\left(\begin{array}{ll}
1 & 0 \\
0 & 1
\end{array}\right)+\sinh \frac{\hbar}{4}\left(\begin{array}{cc}
1 & 0 \\
0 & -1
\end{array}\right) \otimes\left(\begin{array}{cc}
1 & 0 \\
0 & -1
\end{array}\right) .
\end{aligned}
$$

In terms of the parameter $q=e^{-\frac{\hbar}{2}}$, we thus get $\mathcal{R}$ equal to

$a\left(\begin{array}{ll}1 & 0 \\ 0 & 1\end{array}\right) \otimes\left(\begin{array}{ll}1 & 0 \\ 0 & 1\end{array}\right)+b\left(\begin{array}{cc}1 & 0 \\ 0 & -1\end{array}\right) \otimes\left(\begin{array}{cc}1 & 0 \\ 0 & -1\end{array}\right)+c\left(\begin{array}{ll}0 & 1 \\ 0 & 0\end{array}\right) \otimes\left(\begin{array}{ll}0 & 0 \\ 1 & 0\end{array}\right)$,

where

$$
a=\frac{1}{2}\left(q^{-\frac{1}{2}}+q^{\frac{1}{2}}\right), \quad b=\frac{1}{2}\left(q^{-\frac{1}{2}}-q^{\frac{1}{2}}\right), \quad c=q^{\frac{1}{2}}\left(q^{-1}+q\right) .
$$

The identification $M_{2}(\mathbb{C}[[\hbar]]) \otimes M_{2}(\mathbb{C}[[\hbar]])=M_{4}(\mathbb{C}[[\hbar]])$ gives us therefore the following expression

$$
\mathcal{R}=q^{\frac{1}{2}}\left(\begin{array}{cccc}
q^{-1} & 0 & 0 & 0 \\
0 & 1 & q^{-1}-q & 0 \\
0 & 0 & 1 & 0 \\
0 & 0 & 0 & q^{-1}
\end{array}\right)
$$

Fix now the parameter, so $\mathcal{R}$ is considered a $4 \times 4$-matrix with coefficients in $\mathbb{C}$. We will use $\mathcal{R}$ to (re)construct $(\mathcal{A}, \Phi)$ by applying the FRT-construction, [22],[17], which to an arbitrary $R$-matrix gives a co-braided bi-algebra. This inverse construction goes back to the inverse scattering method, [11], and is a way of producing an abundance of $R$-matrices from a given one. Let us briefly explain the main idea of how to construct the bi-algebra (i.e. an algebra with a co-multiplication) from an $R$-matrix $R$. (In fact, the Yang Baxter property of $R$ is only needed to get the co-braiding).

Suppose $R$ is an $n^{2} \times n^{2}$-matrix over $\mathbb{C}$ for some natural number $n$. Let $F$ be the unital universal free algebra over $\mathbb{C}$ generated by elements $t_{i j}, i, j=1, \ldots, n$, and let $T=\left(t_{i j}\right)$ be the 
$n \times n$-matrix with coefficients $t_{i j}$ in this non-commutative algebra $F$. Set $T_{1}=T \otimes I$ and $T_{2}=I \otimes T$ and consider them as elements of the algebra of $n^{2} \times n^{2}$-matrices over $F$ in the usual way. Let $I$ be the ideal of $F$ generated by the $n^{2} n^{2}$ elements $R T_{1} T_{2}-T_{2} T_{1} R$ and form the unital quotient algebra $F / I$, which by construction is just the universal algebra with generators $t_{i j}$ satisfying the relations $R T_{1} T_{2}=T_{2} T_{1} R$. (When $R$ is the identity matrix the algebra $F / I$ is therefore commutative.)

It is straightforward to check that the formulas

$$
\Delta\left(t_{i j}\right)=\sum_{r=1}^{n} t_{i r} \otimes t_{r j}
$$

define a unique co-multiplication $\Delta$ on $F / I$.

Consider now the $R$-matrix $\mathcal{R}$ given above. Here $n=2$ so

$$
T_{1} T_{2}=\left(\begin{array}{cccc}
t_{11} t_{11} & t_{11} t_{12} & t_{12} t_{11} & t_{12} t_{12} \\
t_{11} t_{21} & t_{11} t_{22} & t_{12} t_{21} & t_{12} t_{22} \\
t_{21} t_{11} & t_{21} t_{12} & t_{22} t_{11} & t_{22} t_{12} \\
t_{21} t_{21} & t_{21} t_{22} & t_{22} t_{21} & t_{22} t_{22}
\end{array}\right)=T_{1} \otimes T_{2},
$$

whereas $T_{2} T_{1}$ is the matrix obtained from $T_{1} T_{2}$ by simply commuting all coefficients in $T_{1} T_{2}$. The nontrivial relations stemming from $\mathcal{R} T_{1} T_{2}=T_{2} T_{1} \mathcal{R}$ are thus

$$
\begin{gathered}
t_{11} t_{12}=q t_{12} t_{11}, \quad t_{11} t_{21}=q t_{21} t_{11}, \quad t_{21} t_{12}=t_{12} t_{21}, \\
t_{21} t_{22}=q t_{22} t_{21}, \quad t_{12} t_{22}=q t_{22} t_{12}, \quad t_{22} t_{11}=t_{11} t_{22}+\left(q^{-1}-q\right) t_{21} t_{12} .
\end{gathered}
$$

The same bi-algebra can be obtained as the universal one with generators $t_{i j}$ satisfying the relations $\hat{\mathcal{R}}\left(T_{1} \otimes T_{2}\right)=\left(T_{1} \otimes T_{2}\right) \hat{\mathcal{R}}$, where the matrix $\hat{\mathcal{R}}=\Sigma \mathcal{R}$ is just $\mathcal{R}$ with the two middle rows flipped (of course, the algebra would now be commutative only for the flipped identity matrix). The formulas for the co-multiplication $\Delta$ are exactly as before.

The element $\hat{\mathcal{R}}$ when considered an element in $M_{2}(\mathbb{C}) \otimes$ $M_{2}(\mathbb{C})$, is an endomorphism on $\mathbb{C}^{2} \otimes \mathbb{C}^{2}$. Let $\left\{e_{i}\right\}_{i=1}^{2}$ be the 
basis of $\mathbb{C}^{2}$ giving the basis $\left\{e_{i} \otimes e_{j}\right\}_{i, j=1}^{2}$ of $\mathbb{C}^{2} \otimes \mathbb{C}^{2}$ for which $\hat{\mathcal{R}}$ is expressed as the matrix written above. It is easy to see, [22], that the formulas

$$
\Gamma_{1}\left(e_{i}\right)=\sum_{i=1}^{2} t_{i j} \otimes e_{j}
$$

define a left co-action of the bi-algebra $(F / I, \Delta)$ on $\mathbb{C}^{2}$, i.e. a linear map $\Gamma_{1}: \mathbb{C}^{2} \rightarrow(F / I) \otimes \mathbb{C}^{2}$ such that $\left(\iota \otimes \Gamma_{1}\right) \Gamma_{1}=(\Delta \otimes \iota) \Gamma_{1}$. Y. Manin, [35], took the idea of co-actions even further. Considering instead the vectors $e_{1}$ and $e_{2}$ as generators $x_{1}$ and $x_{2}$ satisfying the relation $x_{1} x_{2}=q x_{2} x_{1}$, he introduced the quantum plane $\mathbb{C}_{q}\left[x_{1}, x_{2}\right]$. By definition it is (dual to) the complex universal algebra with generators $x_{i}$ satisfying the relation $x_{1} x_{2}=q x_{2} x_{1}$. Notice that $x_{1} x_{2}=q x_{2} x_{1}$ is an exponentiated form of Heisenberg's commutation relation $[Q, P]=i \hbar$ for the position $Q$ and momentum $P$ of a particle in a 1-dimensional motion (put $x_{1}=e^{Q}, x_{2}=e^{P}$ and $\left.q=e^{i \hbar}\right)$. When $q=1$ the algebra $\mathbb{C}_{q}\left[x_{1}, x_{2}\right]$ is isomorphic to the algebra of polynomials on $\mathbb{C}^{2}$, and so $\mathbb{C}_{q}\left[x_{1}, x_{2}\right]$ deserves the name quantum plane. Manin used the quantum plane as a device to construct a Hopf algebra. Denote by $T=\left(t_{i j}\right)$ a $2 \times 2$-matrix with indeterminates $t_{i j}$. The rule of the game is now to find relations between these indeterminates such that the formulas

$$
\Gamma\left(x_{i}\right)=\sum_{j=1}^{2} t_{i j} \otimes x_{j}, \quad \Lambda\left(x_{i}\right)=\sum_{j=1}^{2} x_{j} \otimes t_{i j}
$$

define algebra homomorphisms. By universality of $\mathbb{C}_{q}\left[x_{1}, x_{2}\right]$, it is sufficient to require that the relations $\Gamma\left(x_{1}\right) \Gamma\left(x_{2}\right)=q \Gamma\left(x_{2}\right) \Gamma\left(x_{1}\right)$ and $\Lambda\left(x_{1}\right) \Lambda\left(x_{2}\right)=q \Lambda\left(x_{2}\right) \Lambda\left(x_{1}\right)$ should hold, which is the case if and only if the elements $t_{i j}$ satisfy the relations derived from the $R$-matrix $\hat{\mathcal{R}}$ given above. It is automatic in Manin's method that the universal algebra thus obtained forms a bi-algebra with comultiplication $\Delta$ defined by the formulas $\Delta\left(t_{i j}\right)=\sum_{r=1}^{2} t_{i r} \otimes t_{r j}$, and such that the formulas for $\Gamma$ and $\Lambda$ define left and right coactions respectively, of this bi-algebra on the quantum plane. Of 
course the bi-algebra we are talking about here is isomorphic to $(F / I, \Delta)$. Moreover, the (degree-one) subspace of $\mathbb{C}_{q}\left[x_{1}, x_{2}\right]$ linearly spanned by the elements $x_{i}$, is invariant under the left coaction $\Gamma$, and the restriction of $\Gamma$ to this subspace is the same as the left co-action $\Gamma_{1}$ introduced above. A similar result holds for the right co-action $\Lambda$. We denote the bi-algebra $(F / I, \Delta)$ by $\left(M_{q}(2), \Delta\right)$ to emphasize that it comes with co-actions on the quantum plane.

Now $\left(M_{q}(2), \Delta\right)$ is not a Hopf algebra, because (although the formulas $\varepsilon\left(t_{i j}\right)=\delta_{i j}$ define a co-unit) no antipode can be defined which is consistent with the relations for the generators $t_{i j}$. To get consistency, one needs to add (in a compatible way) more elements and relations such that the matrix $T$ becomes invertible. In the classical limit this is done by requiring the determinant to be non zero. The element $D_{q}(T)=t_{11} t_{22}-q t_{12} t_{21}$ has properties resembling those of a determinant in that it is multiplicative (i.e. $\Delta\left(D_{q}(T)\right)=D_{q}(T) \otimes D_{q}(T)$ and $\left.\varepsilon\left(D_{q}(T)\right)=1\right)$, and so is called the quantum determinant of $T$. It can be shown that it generates the center of the algebra $M_{q}(2)$.

The invertibility of $T$ amounts to requiring the quantum determinant to be invertible, which can be done by adding to the algebra $M_{q}(2)$ the new generator $D^{-1}$ commuting with all the generators $t_{i j}$ and satisfying the relations $D^{-1} D_{q}(T)=D_{q}(T) D^{-1}=$ $I$, which in the limit $q \rightarrow 1$ say that the determinant is nonzero. The bi-algebra $\left(G L_{q}(2), \Delta\right)$ thus obtained, with $\Delta$ extended such that $\Delta\left(D^{-1}\right)=D^{-1} \otimes D^{-1}$, is a Hopf algebra with co-inverse $S$ given by the quantum Cramer rule

$$
\begin{array}{cc}
S\left(t_{11}\right)=D^{-1} t_{22}, & S\left(t_{12}\right)=-q^{-1} D^{-1} t_{12}, \\
S\left(t_{21}\right)=-q D^{-1} t_{21}, & S\left(t_{22}\right)=D^{-1} t_{11}
\end{array}
$$

The Hopf algebra $\left(S L_{q}(2), \Delta\right)$ obtained by setting $D^{-1}=I$ in the above formulas, is referred to as the quantum special linear group. It is clearly the surjective image of the Hopf algebra $\left(G L_{q}(2), \Delta\right)$, which is usually called the quantum linear group. Of course Manin's construction works in more generality, and trivially includes the examples $\left(S L_{q}(n), \Delta\right)$ co-acting on a the $n$ dimensional quantum plane, [35]. 
There is a *-operation on the algebra $S L_{q}(2)$ given by

$$
t_{11}^{*}=t_{22}, \quad t_{12}^{*}=-q t_{21},
$$

which follows by universality of $S L_{q}(2)$. It makes $\Delta$ into a *-homomorphism, and it should not come as a surprise that the Hopf *-algebra we thus get is isomorphic to $(\mathcal{A}, \Phi)$ under the map which sends $t_{i j}$ to $u_{i j}$.

As a matter of fact, we may obtain every irreducible unitary co-representation of $(\mathcal{A}, \Phi)$ using $\Gamma$. Namely, let $\mathbb{C}_{q}^{n}\left[x_{1}, x_{2}\right]$ denote the finite-dimensional subspace of $\mathbb{C}_{q}\left[x_{1}, x_{2}\right]$ consisting of the homogeneous elements of degree $n \in\{0,1,2, \ldots\}$. It is easily checked that the space $\mathbb{C}_{q}^{n}\left[x_{1}, x_{2}\right]$ is invariant under $\Gamma$, and so the restriction $\Gamma_{n}$ of $\Gamma$ to $\mathbb{C}_{q}^{n}\left[x_{1}, x_{2}\right]$ yields a unitary co-representation of $(\mathcal{A}, \Phi)$. One may show that the collection $\left(\Gamma_{n}\right)_{n=0}^{\infty}$ form a complete family of pairwise inequivalent irreducible unitary corepresentations of $(\mathcal{A}, \Phi)$. Clearly, $\Gamma_{1}$ corresponds to the fundamental co-representation $u$.

We have thus completed the second way of constructing $(\mathcal{A}, \Phi)$ from the Hopf ${ }^{*}$-algebra $\left(U_{\hbar}(s l(2)), \hat{\Delta}_{\hbar},{ }^{*}\right)$. Note that we needed the fundamental representation to get $\mathcal{R}$ from the universal $R$-matrix, but we could have used the matrix $\mathcal{R}$ as a staring point. Also Manin's method does not rely on the representation theory of $\left(U_{\hbar}(\operatorname{sl}(2)), \hat{\Delta}_{\hbar},{ }^{*}\right)$; it is the quantum way of producing groups as symmetry objects. There are two other *-operations on $S L_{q}(2)$ which correspond to the important real forms $S L_{q}(2, \mathbb{R})$ and $S U_{q}(1,1)$, but none of them are compact. Treating them as locally compact $\mathrm{C}^{*}$-algebraic quantum groups turns out to be very difficult.

Finally we make some remarks on how to get from the 'function algebra ' $(\mathcal{A}, \Phi)$ (or more precisely $(\mathcal{A}, u)$ ) to the Jimbo algebra $\left(U_{q}(s l(2)), \hat{\Delta},{ }^{*}\right)$. Classically this amounts to going from the Lie group $S U(2)$ to the Lie algebra $s u(2)$, which is achieved by using the smooth structure on the manifold $S U(2)$ to form the Lie algebra of smooth left-invariant vector fields. As mentioned before, compact matrix pseudo groups play the role of quantum 
Lie groups and thus suggest that some smooth structure is around which in turn should determine a reasonable quantum Lie algebra.

However, things do not always run smoothly. Let us consider the quantum $S U(2)$ case, and look for what are the obvious candidates for 'left-invariant vector fields' . Consider the generators $e, f, k, k^{-1}$ for $U_{q}(s l(2))$ and form the elements $X_{i} \in \operatorname{End}(\mathcal{A})$ given by $X_{1}=\hat{e}, X_{2}=\hat{f}$ and $X_{3}=\left(\hat{k}-k^{-1}\right)$. Clearly

$$
X_{i}(a b)=X_{i}(a) \hat{k}^{-1}(b)+\hat{k}(a) X_{i}(b), \quad \hat{k}^{ \pm}(a b)=\hat{k}^{ \pm}(a) \hat{k}^{ \pm}(b)
$$

for all $a, b \in \mathcal{A}$, so they are not derivations, but rather twisted derivations. Hence the generators for the universal enveloping algebra $U_{q}(s l(2))$ show up as twisted derivations which certainly do not form a Lie algebra (in fact, the only decent derivation on $\mathcal{A}$ is formally $\hat{H}$ ). But clearly the Hopf *-algebra they generate (together with $\hat{k}$ ) inside $\operatorname{End}(\mathcal{A})$ is dual to the Hopf *-algebra $(\mathcal{A}, \Phi)$ with bilinear form given by

$$
\langle X, a\rangle=\varepsilon(X(a)),
$$

where $\varepsilon$ is the co-unit of $(\mathcal{A}, \Phi)$.

One is thus led to consider twisted derivations. The first step in that direction was taken by Woronowicz, [79]. Inspired by the program set out by Alain Connes, [7], to develop noncommutative differential geometry on arbitrary quantum spaces, Woronowicz constructed a 3-dimensional differential calculus on quantum $S U(2)$ using differential forms derived from twisted derivations. The twistedness is then hidden in nontrivial actions of the algebra $\mathcal{A}$ on the module of differential forms. He applied the differential calculus successfully to find all the finite-dimensional irreducible unitary co-representations of quantum $S U(2)$ by reducing the problem to classifying their infinitesimal generators which in turn induced the desired (global) co-representations.

Later, [76], Woronowicz developed a general framework for differential calculus on compact matrix pseudo groups again using differential forms. Important in Woronowicz' framework is the notion of bi-covariance, which allows differential forms to be 
translated by left and right quantum group actions, so left and right invariant differential forms can be defined. The exigency of this notion excluded his previous differential calculus defined on quantum $S U(2)$, and the idea of simple twisted derivations producing (in a fairly direct way) the differential forms had to be abandoned. It turns out that no 3-dimensional bi-covariant calculus can be defined on quantum $S U(2)$, [53], (Woronowicz' example is only left covariant). However, Woronowicz defined a 4-dimensional bi-covariant calculus $D_{+}$for quantum $S U(2)$. Also he established a one-to-one correspondence between (first order) bi-covariant calculi and certain right ideals of the algebras of regular functions on the compact matrix pseudo groups, namely as the annihilator of certain generalized twisted derivations. The right ideal $\mathcal{R}_{+}$corresponding to $D_{+}$is given by

$$
\mathcal{R}_{+}=\left\{a \in \mathcal{A} \mid k e(a)=k f(a)=k\left(k-k^{-1}\right)(a)=C(a)=0\right\},
$$

where $C$ is the quantum Casimir element defined as

$$
C=e f+f e+c\left(k-k^{-1}\right)^{2}
$$

with $c=\left(q+q^{-1}\right) /\left(q-q^{-1}\right)^{2}$. One should notice that $C$ is classically a second order differential operator.

A serious defect with the theory of bi-covariant differential calculi on general compact matrix pseudo groups, is that no canonical construction has been suggested for them, probably since at the present stage, too little is known about the smooth structure on these quantum groups. Furthermore, the property of uniqueness is violated, for instance, there are (exactly) two nonisomorphic 4-dimensional bi-covariant calculi on quantum $S U(2)$, [53]. For classification of an important class of bi-covariant differential calculi on compact matrix pseudo groups dual to the Jimbo algebra deformations of complex simple Lie algebras, [49]. The uniqueness is violated to the extreme when it comes to the more general notions of left (or right) covariant calculi; on quantum $S U(2)$ there are infinitely many non-isomorphic ones, [56]. We think it is fair to say that at the moment (although fragments are 
there) no satisfactory theory for differential structures on quantum groups has been developed that is as powerful in the structure theory of quantum groups as Lie algebras has been for the structure theory of Lie groups. It should be mentioned though, that P. Schupp, P. Watts and B. Zumino have found a partial solution to the problem, in that they have given a method for constructing bi-covariant differential calculi for quantum groups with $R$-matrices (or quasi-triangular Hopf algebras), [50].

\section{Locally Compact Quantum Groups}

The general theory of locally compact quantum groups is much less understood than the theories of compact, discrete and algebraic quantum groups discussed in the previous sections. An understanding of the relation between these non-compact $\mathrm{C}^{*}$-algebraic quantum groups and the quantized universal algebras of Drinfeld and Jimbo is absent at the present stage. It is only now, after a generation of research on the subject, from which a clear enough picture of the general case has emerged, that a reasonable definition of a locally compact quantum group can be given. The reason for this is, of course, the highly nontrivial techniques that have been required to overcome the conceptual difficulties encountered in such a general and yet restricted theory. Obviously, proving results from such a general definition is technically challenging and examples are hard to construct and investigate. Consequently, only a few new examples have been constructed so far, since as a rule, it takes at least a year of progressive work to develop a single new interesting example of a locally compact quantum group (such as $S U(1,1)$ and $a x+b$ ).

In this section we first review the classical example of a locally compact group in order to motivate the definition of a locally compact quantum group. After having provided some terminology, we state the definition, and we finish by looking briefly at the example of quantum $\mathrm{E}(2)$ studied by Woronowicz (see $[74],[75],[65])$.

Suppose $G$ is a locally compact group. As in the simpler case of Section 3 (part I), it is possible to associate two different 
locally-compact-quantum-group-objects to this group (as already mentioned, the term 'locally compact quantum group' will be given a precise meaning later on).

Example 1. Let $C_{0}(G)$ denote the set of continuous functions on $G$ which vanish at infinity. We turn $C_{0}(G)$ into a commutative $\mathrm{C}^{*}$-algebra by defining all the operations in a pointwise manner and using the supremum norm. This $\mathrm{C}^{*}$-algebra is unital if and only if $G$ is compact. By Gelfand's theorem we know that all commutative $\mathrm{C}^{*}$-algebras arise from locally compact spaces in this way. The multiplier algebra $M\left(C_{0}(G)\right)$ is nothing else but the $\mathrm{C}^{*}$-algebra $C_{b}(G)$ of bounded continuous functions on $G$, and the minimal tensor product $C_{0}(G) \otimes C_{0}(G)$ can be naturally identified with $C_{0}(G \times G)$. Hence $M\left(C_{0}(G) \otimes C_{0}(G)\right)$ can be identified with $C_{b}(G \times G)$

We translate the group structure on $G$ to $C_{0}(G)$ by defining the non-degenerate ${ }^{*}$-homomorphisms $\Delta, \varepsilon, S$ as follows:

- $\Delta: C_{0}(G) \rightarrow M\left(C_{0}(G) \otimes C_{0}(G)\right)$, where $\Delta(f)(s, t)=f(s t)$ for all $f \in C_{0}(G)$ and $s, t \in G$.

- $\varepsilon: C_{0}(G) \rightarrow \mathbb{C}$ and $S: C_{0}(G) \rightarrow C_{0}(G)$, where $\varepsilon(f)=f(e)$ and $S(f)(s)=f\left(s^{-1}\right)$ for all $f \in C_{0}(G)$ and $s \in G$.

The maps $\Delta, S$ and $\varepsilon$ determine a quantum group structure on $C_{0}(G)$.

Example 2. Another way of introducing a quantum-grouplike-object, is via the group $\mathrm{C}^{*}$-algebra construction. It is more involved than the above construction. One starts by fixing a left Haar measure $\mu$ on $G$ and considers the normed space $L^{1}(G)$ of integrable functions on $G$ with respect to $\mu$, where the norm is the ordinary $L^{1}$-norm. Next, it is customary to turn $L^{1}(G)$ into a *algebra by introducing the convolution product $*$ and appropriate *-operation ${ }^{\circ}$ on $L_{1}(G)$ :

- $(f * g)(t)=\int f(s) g\left(s^{-1} t\right) d \mu(s)$ for all $f, g \in L^{1}(G)$ and almost all $t \in G$,

- $f^{\circ}(t)=\delta(t)^{-1} \overline{f\left(t^{-1}\right)}$ for all $f \in L^{1}(G)$ and almost all $t \in G$,

where $\delta$ denotes the modular function of the locally compact group $G$ which connects the left and the right Haar measure on $G$. It 
should be stressed that $L^{1}(G)$ is not a $\mathrm{C}^{*}$-algebra, only a Banach *-algebra. One obtains a $\mathrm{C}^{*}$-algebra by putting a $\mathrm{C}^{*}$-norm on this *-algebra and completing $L^{1}(G)$ with respect to this $\mathrm{C}^{*}$-norm.

A possible way of doing this is by taking the universal enveloping $\mathrm{C}^{*}$-algebra of $L^{1}(G)$. The $\mathrm{C}^{*}$-algebra one obtains in this way is denoted by $C^{*}(G)$ and is referred to as the universal dual of $G$. But we can also construct a second (in general different) $\mathrm{C}^{*}$-algebra by using the left regular representation of $G$. The left regular representation $\lambda_{l}$ of $G$ is a unitary representation of $G$ acting on the space of square integrable functions $L^{2}(G)$, and is defined by the formula $\left(\lambda_{l}(s) g\right)(t)=g\left(s^{-1} t\right)$ for all $g \in L^{2}(G)$ and $s, t \in G$. Now $\lambda_{l}$ has a unique extension to the left regular *-representation $\lambda$ of $L^{1}(G)$ on $L^{2}(G)$, defined such that $\lambda(f)=\int f(s) \lambda_{l}(s) d \mu(s)$ for all $f \in L^{1}(G)$, where the integral is to be understood as a Bochner integral taken in the strong topology of $B(H)$. One may prove that $\lambda: L^{1}(G) \rightarrow B\left(L^{2}(G)\right)$ is a faithful ${ }^{*}$-representation. Define $C_{r}^{*}(G)$ to be the closure of $\lambda\left(L^{1}(G)\right)$ in $B(H)$. The $\mathrm{C}^{*}$-algebra $C_{r}^{*}(G)$ is referred to as the reduced dual of $G$. The image of the map $\lambda_{l}: G \rightarrow B(H)$ is contained in the multiplier algebra $M\left(C_{r}^{*}(G)\right)$.

It is possible (but is beyond the scope of this survey) to prove the existence of

- a unique non-degenerate ${ }^{*}$-homomorphism $\Delta: C_{r}^{*}(G) \rightarrow$ $M\left(C_{r}^{*}(G) \otimes C_{r}^{*}(G)\right)$ such that $\Delta\left(\lambda_{r}(s)\right)=\lambda_{r}(s) \otimes \lambda_{r}(s)$ for all $s \in G$,

- a unique *-anti-automorphism $S: C_{r}^{*}(G) \rightarrow C_{r}^{*}(G)$ such that $S\left(\lambda_{r}(s)\right)=\lambda_{r}\left(s^{-1}\right)$ for all $s \in G$.

Let us now look at the case where $G$ is abelian. Classical group theory tells us how to construct the dual group $\hat{G}$. As a set, $\hat{G}$ is the set of all continuous group characters on $G$ taking values in the unit circle. The group multiplication on $\hat{G}$ is just the pointwise multiplication of two characters. The topology of $\hat{G}$ is the compact-open topology, in which a net of elements in $\hat{G}$ converges to an element in $\hat{G}$ if it converges uniformly to this element on each compact subset of $G$. In this way, $\hat{G}$ is endowed with the structure of a commutative locally compact group. The 
celebrated Pontryagin duality theorem says that the mapping $\theta$ : $G \rightarrow \hat{\hat{G}}$ defined by $\theta(s)(\omega)=\omega(s)$ for all $\omega \in \hat{G}$ and $s \in G$, is a group isomorphism and a homeomorphism.

It is possible to identify $C_{r}^{*}(G)$ with $C_{0}(\hat{G})$ through a ${ }^{*}$-isomorphism $\pi: C_{r}^{*}(G) \rightarrow C_{0}(\hat{G})$ defined so that $\pi(\lambda(f))(\omega)=$ $\int f(s) \omega(s) d \mu(s)$ for all $f \in C_{r}^{*}(G)$ and $\omega \in \hat{G}$. It turns out that this map $\pi$ is compatible with the quantum group structure, that is $(\pi \otimes \pi) \Delta=\Delta \pi$ and $S \pi=\pi S$. In other words, $C_{r}^{*}(G)$ and $C_{0}(\hat{G})$ are isomorphic as quantum groups.

This discussion holds for abelian groups but fails for nonabelian ones. It is impossible to define (by a general construction) an appropriate dual locally compact group which encodes essentially all the information about the original locally compact group. However, one can prove that the reduced dual $\mathrm{C}^{*}$-algebra $C_{r}^{*}(G)$ encodes (as a quantum group) all information about $G$. But if $G$ is not abelian, this $\mathrm{C}^{*}$-algebra $C_{r}^{*}(G)$ is noncommutative and cannot arise as $\left(C_{0}(H), \Delta\right)$, for some locally compact group $H$.

In the beginning of the quantum group era (within the von Neumann algebra or $\mathrm{C}^{*}$-algebra framework), people tried to overcome this problem by enlarging the category of groups to a category of 'quantum groups' which contained the groups and reduced group duals, and which allowed for a dual construction for which a Pontryagin duality theorem held (i.e. the dual of the dual is isomorphic to the original quantum group). These considerations where the main reasons for developing the theory of Kac algebras. After pioneering work by T. Tannaka, M. G. Krein, G. I. Kac and M. Takesaki, among others, the final solution was developed independently by M. Enock \& J. -M. Schwartz (see [14] for a full exposition) and by Kac \& L. L. Vainerman ([58], [57]) in the seventies. The theory of Kac algebras is formulated in the von Neumann algebra framework.

For quite a time, the main disadvantage of this theory lay in the fact that there was a lack of interesting examples aside from the groups and group duals. We will not state the definition of a Kac algebra but we will comment on the class of these algebras 
after we have given the general definition of a quantum group. It turns out that the general definition of a quantum group is simpler than that of a Kac algebra but Kac algebras satisfy some extra, nice properties that make them easier to handle.

Woronowicz constructed in [79] a quantized version of $S U(2)$, an object that has all the right properties to deserve being called a compact quantum group, but that does not fit into the framework of Kac algebras. In subsequent papers ([78],[72]), Woronowicz developed an axiom scheme for compact quantum groups. In contrast to the Kac algebra theory, quantum SU(2) fits into the category of compact quantum groups.

The main difference between compact Kac algebras and compact quantum groups lies in the fact that the antipode of the Kac algebra is a (bounded) automorphism while in the approach of Woronowicz, it may be unbounded (this is the case for quantum $S U(2)$ ). However, it should be pointed out that a lot of the ideas and proofs from the theory of Kac algebras can easily be generalized to the quantum group setting.

It was E. Kirchberg ([24]) who proposed a generalized axiom scheme for quantum groups, where the antipode is unbounded but can be decomposed as a product of an automorphism and an unbounded operator generated by a one-parameter group. This decomposition is called the polar decomposition of the antipode, and it appeared also in the compact case, [72]. The general case was treated in the von Neumann algebra setting, [37], by Masuda and Nakagami, who also suggested a definition of a locally compact quantum group and proved a duality result. The main problem of their proposed definition lies in the complexity of the axioms. In [30], the first author and S. Vaas propose a much simpler definition of a locally compact quantum group (in its reduced form). We will probe a little bit deeper into this definition in the next part of this section.

Before stating the definition of a locally compact quantum group, we need some extra terminology concerning weights on $\mathrm{C}^{*}$-algebras. The most important objects associated to a locally 
compact group are its Haar measures. So it is no big surprise that also in the quantum group setting equally fundamental roles are played by the proper generalizations of these measures. Their importance in the more general setting is even more pronounced, because - to the present - their existence is an axiom in the definition of a quantum group. This might seem unsettling for those who insist on deducing the existence of the Haar measures from the axioms of a locally compact group. We should point out, however, that although existence is assumed, essential uniqueness is proved, and, as is well known from the classical case, when working with examples the Haar measures tend to suggest themselves (say after calculating a couple of Jacobi determinants). Therefore existence of the Haar measure is not really an issue in practice. Finally, is not the theory of locally compact groups the study of topological groups with Haar measures, just as amenable groups is the study of groups with bounded invariant measures? It turns out that most properties of a locally compact quantum group can be deduced from the existence of generalized Haar measures.

The usual way to generalize measures (or rather their integrals) on locally compact spaces is to use weights on von Neumann algebras or, more generally, on $\mathrm{C}^{*}$-algebras. The formal definition of a weight is as follows:

Consider a $\mathrm{C}^{*}$-algebra $A$ and a function $\varphi: A^{+} \rightarrow[0, \infty]$ such that:

1. $\varphi(x+y)=\varphi(x)+\varphi(y)$ for all $x, y \in A^{+}$,

2. $\varphi(r x)=r \varphi(x)$ for all $x \in A^{+}$and $r \in[0, \infty[$ (where $0(+\infty)=0)$.

We call $\varphi$ a weight on $A$. The weight $\varphi$ is called faithful if $\varphi(x)=0$ $\Leftrightarrow x=0$ for all $x \in A^{+}$. Denote the set of positive integrable elements of $\varphi$ by $\mathcal{M}_{\varphi}^{+}$, and the set of all integrable elements by $\mathcal{M}_{\varphi}$. More precisely, $\mathcal{M}_{\varphi}^{+}=\left\{x \in A^{+} \mid \varphi(x)<\infty\right\}$, and $\mathcal{M}_{\varphi}$ is the linear span of $\mathcal{M}_{\varphi}^{+}$. There exists a unique linear mapping $\psi$ on $\mathcal{M}_{\varphi}$ which extends $\varphi$, and we put $\varphi(x):=\psi(x)$ for all $x \in \mathcal{M}_{\varphi}$.

In order to render weights useful, we have to impose a continuity condition on them. The relevant continuity condition is the usual lower semi-continuity as a function from $A^{+}$to $[0, \infty]$. 
Loosely speaking, this boils down to requiring the weight to satisfy the Lemma of Fatou (lower semi-continuity also implies some monotone convergence properties).

A truly non-commutative phenomenon is the KMS property for weights. Although the $\mathrm{C}^{*}$-algebra may be non-commutative, the KMS condition gives some control over the non-commutativity under the weight. In order to make this more precise, we need the notion of a one-parameter groups and its analytic extension.

Let $\alpha: \mathbb{R} \rightarrow \operatorname{Aut}(A)$ be a mapping such that:

1. $\alpha_{s} \alpha_{t}=\alpha_{s+t}$ for all $t \in \mathbb{R}$.

2. the function $\mathbb{R} \rightarrow A: t \rightarrow \alpha_{t}(a)$ is norm-continuous for all $a \in A$,

where $\operatorname{Aut}(A)$ denotes the set of all automorphisms of the $\mathrm{C}^{*}$-algebra $A$.

We call $\alpha$ a norm-continuous one-parameter group on $A$. There is a standard way to define for every $z \in \mathbb{C}$, a closed, densely-defined, multiplicative, linear operator $\alpha_{z}$ in $A$ :

- The domain $D\left(\alpha_{z}\right)$ of $\alpha_{z}$ is by definition the set of elements $x \in A$ such that there exists a function $f$ (depending on $x$ ) from the strip $S(z)=\{y \in \mathbb{C} \mid \operatorname{Im} y \in[0, \operatorname{Im} z]\}$ to $A$ such that

1. $f$ is continuous on $S(z)$,

2. $f$ is analytic on the interior of $S(z)$,

3. $\alpha_{t}(x)=f(t)$ for every $t \in \mathbb{R}$.

- For $x \in D\left(\alpha_{z}\right)$, the function $f$ is unique, and we define $\alpha_{z}(x)$ to be $f(z)$.

Now consider a faithful weight $\varphi$ on $A$. It is called a $K M S$ weight if there exists a norm-continuous one-parameter group $\sigma$ on $A$ such that:

1. $\varphi$ is invariant under $\sigma$, that is, $\varphi \sigma_{t}=\varphi$ for every $t \in \mathbb{R}$,

2. $\varphi\left(a^{*} a\right)=\varphi\left(\sigma_{\frac{i}{2}}(a) \sigma_{\frac{i}{2}}(a)^{*}\right)$ for every $a \in D\left(\sigma_{\frac{i}{2}}\right)$.

If $\varphi$ is a KMS-weight (this is not automatically true for every lower semi-continuous faithful weight), then this one-parameter group $\sigma$ is unique and it is called the modular group of $\varphi$. Our 
previous remark about the control of non-commutativity under the weight, can be given a precise meaning as follows: for $a \in$ $D\left(\sigma_{-i}\right)$ and $x \in \mathcal{M}_{\varphi}$, the elements $a x$ and $x \sigma_{-i}(a)$ belong to $\mathcal{M}_{\varphi}$ and $\varphi(a x)=\varphi\left(x \sigma_{-i}(a)\right)$.

This KMS condition is really the key result that allows one to develop a generalized non-commutative measure theory that parallels classical measure theory (For instance, the RadonNikodym Theorem has a generalization to weights in the von Neumann algebra framework).

We have now gathered enough material to formulate the definition of a locally compact quantum group.

Definition 7.1 Consider a $C^{*}$-algebra $A$ and a non-degenerate *-homomorphism $\Delta: A \rightarrow M(A \otimes A)$ such that:

- $(\Delta \otimes \iota) \Delta=(\iota \otimes \Delta) \Delta$.

- $A=\left[(\omega \otimes \iota) \Delta(a) \mid \omega \in A^{*}, a \in A\right]=\left[(\iota \otimes \omega) \Delta(a) \mid \omega \in A^{*}, a \in\right.$ A].

Assume moreover the existence of:

1. A faithful KMS-weight $\varphi$ on $(A, \Delta)$ such that $\varphi((\omega \otimes$ ८) $\Delta(x))=\varphi(x) \omega(1)$ for $\omega \in A_{+}^{*}$ and $x \in \mathcal{M}_{\varphi}^{+}$.

2. A KMS-weight $\psi$ on $(A, \Delta)$ such that $\psi((\iota \otimes \omega) \Delta(x))=$ $\psi(x) \omega(1)$ for $\omega \in A_{+}^{*}$ and $x \in \mathcal{M}_{\psi}^{+}$.

Then we call $(A, \Delta)$ a reduced $C^{*}$-algebraic quantum group.

The equality in condition 1 of this definition is called the left invariance of the weight $\varphi$. An important property of quantum groups is uniqueness of left-invariant weights: any lower semicontinuous left-invariant weight $\Phi$ on $(A, \Delta)$ is proportional to $\varphi$ (that is, $\exists c \in \mathbb{R}$ such that $\Phi=c \varphi$ ). It should be noted that it is possible to relax the KMS condition somewhat and still get an equivalent definition. Similar remarks apply to right invariant weights.

As already mentioned, the main drawback of this definition is the assumption of the existence of the left and right invariant weights (including their KMS properties), which is in sharp contrast to the compact and discrete cases. So far no one 
has been able to formulate a general definition of a locally compact quantum group without assuming the existence of invariant weights.

The prefix 'reduced' used in the definition is added because we require the left-invariant weight $\varphi$ to be faithful. However, given any ' $\mathrm{C}^{*}$-algebraic quantum group' , one can associate to it a reduced $\mathrm{C}^{*}$-algebraic quantum group which is essentially equivalent to the original $\mathrm{C}^{*}$-algebraic quantum group. For more on this see [27], where universal $\mathrm{C}^{*}$-algebraic quantum groups are defined.

From these axioms, one can construct (but this is highly nontrivial) the antipode $S$ which is a closed, generally unbounded, operator that is only densely defined. The unboundedness is controlled by the existence of a unique (bounded) ${ }^{*}$ anti-automorphism $R$ on $A$ and a unique norm-continuous oneparameter group $\tau$ on $A$ such that

- $R^{2}=\iota$,

- $R$ and $\tau$ commute,

- $S=R \tau_{-\frac{i}{2}}$.

The pair $(R, \tau)$ is called the polar decomposition of $S$. The *-antiautomorphism $R$ is called the unitary antipode of $(A, \Delta)$ and the one-parameter group $\tau$ is called the scaling group of $(A, \Delta)$. A Kac algebra is nothing else but a locally compact quantum group for which $\tau_{t}=\iota$ for all $t \in \mathbb{R}$, or equivalently $S=R$.

The unitary antipode anti-commutes with $\Delta$ : that is, $\chi(R \otimes$ $R) \Delta=\Delta R$, where $\chi$ denotes the flip-automorphism extended to $M(A \otimes A)$. This means in particular, that $\varphi R$ is a faithful right invariant KMS-weight on $A$. It should be pointed out that $\psi$ is needed in the construction of $S, R$ and $\tau$. Also, $\Delta \sigma_{t}=\left(\tau_{t} \otimes \sigma_{t}\right) \Delta$ and $\Delta \tau_{t}=\left(\tau_{t} \otimes \tau_{t}\right) \Delta$ for all $t \in \mathbb{R}$.

The role of the $L^{2}$-space of a measure is played by the GNSrepresentation associated to the weight $\varphi$. This is a triple $(H, \iota, \Lambda)$, where: 
- $H$ is a Hilbert space,

- $\Lambda$ is a linear map from $\mathcal{N}_{\varphi}$ into $H$ such that:

1. $\Lambda\left(\mathcal{N}_{\varphi}\right)$ is dense in $H$,

2. $\langle\Lambda(a), \Lambda(b)\rangle=\varphi\left(b^{*} a\right)$ for every $a, b \in \mathcal{N}_{\varphi}$,

- $\pi$ is a *-representation of $A$ on $H$ such that $\pi(a) \Lambda(b)=\Lambda(a b)$ for every $a \in A$ and $b \in \mathcal{N}_{\varphi}$.

Here $\mathcal{N}_{\varphi}$ denotes the set of all square-integrable elements of $\varphi$ in A,

$$
\mathcal{N}_{\varphi}=\left\{x \in A \mid \varphi\left(x^{*} x\right)<\infty\right\}
$$

and $\langle\cdot, \cdot\rangle$ is the inner product on $H$.

The multiplicative unitary $W$ of $(A, \Delta)$ is by definition the unique unitary element in $B(H \otimes H)$ such that $W(\Lambda \otimes \Lambda)(\Delta(b)(a \otimes$ $1))=\Lambda(a) \otimes \Lambda(b)$ for all $a, b \in \mathcal{N}_{\varphi}$. The operator $W$ satisfies the pentagonal equation

$$
W_{12} W_{13} W_{23}=W_{23} W_{12}
$$

It encodes the structure of $(A, \Delta)$ in the following way:

- $A=\left[(\iota \otimes \omega)(W) \mid \omega \in B_{0}(H)^{*}\right]$,

- $\Delta(x)=W^{*}(1 \otimes x) W$ for all $x \in A$.

Also, $W$ can be used to define the dual of $(A, \Delta)$. Set:

- $\hat{A}=\left[(\omega \otimes \iota)(W) \mid \omega \in B_{0}(H)^{*}\right]$,

- $\hat{\Delta}(x)=\Sigma W(x \otimes 1) W^{*} \Sigma$ for all $x \in \hat{A}$,

where $\Sigma$ denotes the flip map on $H \otimes H$. One can prove that $(\hat{A}, \hat{\Delta})$ is again a reduced $\mathrm{C}^{*}$-algebraic quantum group and we call it the reduced dual of $(A, \Delta)$. Furthermore, the reduced dual of $(\hat{A}, \hat{\Delta})$ is canonically isomorphic to $(A, \Delta)$ as a quantum group, a profound generalization of Pontryagin's duality theory for abelian locally compact groups.

The first example of non-compact quantum groups considered - they are those for which the underlying $\mathrm{C}^{*}$-algebra 
is non-unital - were quantum deformations of the group $E(2)$ of motions of the plane which preserve distance and orientation (i.e. rotations, translations and compositions thereof). This example was discovered and developed by Woronowicz (see $[74],[75])$. Baaj should also be mentioned ([3]), mainly in connection with the left Haar weight and the non-regularity of quantum $E(2)$. Van Daele has collaborated with Woronowicz in treating its dual $([65])$.

Let us identify the real plane $\mathbb{R}^{2}$ with the complex plane $\mathbb{C}$, so that a rotation corresponds to multiplication by a complex number of modulus 1 and a translation corresponds to adding a complex number.

We can therefore regard $E(2)$ as a subgroup of the group of invertible $2 \times 2$ matrices over $\mathbb{C}$ :

$$
E(2)=\left\{\left(\begin{array}{ll}
z & x \\
0 & 1
\end{array}\right) \mid z, x \in \mathbb{C} \text { and }|z|=1\right\}
$$

Here an element $c \in \mathbb{C}$ corresponds to the column $\left(\begin{array}{l}c \\ 1\end{array}\right)$, and the matrices from $E(2)$ act on these columns by left multiplication.

Define the continuous coordinate functions $n, u: E(2) \rightarrow \mathbb{C}$ by

$$
n\left(\begin{array}{cc}
z & x \\
0 & 1
\end{array}\right)=x \quad \text { and } \quad u\left(\begin{array}{cc}
z & x \\
0 & 1
\end{array}\right)=z
$$

for all $x, z \in \mathbb{C}$. Notice that $u$ is a unitary element of $M\left(C_{0}(E(2))\right.$ and that $n$ does not belong to this multiplier algebra. It is only 'affiliated' to the non-unital C*-algebra $C_{0}(E(2))$. The two elements $n$ and $u$ do not generate the $\mathrm{C}^{*}$-algebra $C_{0}(E(2)$ in the ordinary sense (as said, they do not even belong to it), but they determine it in the sense that it is generated by $\{u\} \cup\{f \circ n \mid f \in$ $\left.C_{0}(\mathbb{C})\right\}$.

Using the group multiplication on $E(2)$ to define a comultiplication $\Delta: C_{0}(E(2)) \rightarrow C_{b}(E(2) \times E(2))$ one gets $\Delta(u)=u \otimes u$ and $\Delta(n)=u \otimes n+n \otimes 1$ (it should be pointed out 
that it is possible to extend a non-degenerate *-homomorphism, such as $\Delta$, to the set of affiliated elements).

Just as in the case of quantum $S U(2)$ one would like to construct quantum $E(2)$ by quantizing or deforming the $\mathrm{C}^{*}$-algebra $C_{0}(E(2))$. Therefore we fix a deformation parameter $\left.\nu \in\right] 1, \infty[$. We will now discuss the two main problems in performing this deformation.

1. The construction of the $\mathrm{C}^{*}$-algebra:

Similar to the quantum $\mathrm{SU}(2)$ case, one would like to define the $\mathrm{C}^{*}$-algebra $A\left(E_{\nu}(2)\right)$ to be the $\mathrm{C}^{*}$-algebra 'generated' by a unitary element $u$ in $M\left(A\left(E_{\nu}(2)\right)\right)$, a normal element $n$ 'affiliated' to $A\left(E_{\nu}(2)\right)$ that do not commute but that satisfy the commutation relation $u^{*} n u=\nu n$. This $\mathrm{C}^{*}$-algebra should be determined by the following universality property:

Let $H$ be a Hilbert space, $U$ a unitary element in $B(H)$ and $N$ a normal closed operator in $H$ such that $U^{*} N U=\nu N$. Then there exists a unique non-degenerate ${ }^{*}$-homomorphism $\theta$ : $A\left(E_{\nu}(2)\right) \rightarrow B(H)$ such that $\theta(u)=U$ and $\theta(n)=N$.

There is no general principle which allows one to construct such $\mathrm{C}^{*}$-algebras by such 'generators' and 'relations'. In all cases one has to come up with a $\mathrm{C}^{*}$-algebra that satisfies the appropriate universality property. In general, such a universal $\mathrm{C}^{*}$-algebra does not have to exist.

2. Defining the co-multiplication:

We would like to define a co-multiplication $\Delta: A\left(E_{\nu}(2)\right) \rightarrow$ $M\left(A\left(E_{\nu}(2)\right) \otimes A\left(E_{\nu}(2)\right)\right)$ such that $\Delta(u)=u \otimes u$ and $\Delta(n)=$ $u \otimes n \dot{+} n \otimes 1$. There are some major problems with this last expression. What do we actually mean by $u \otimes n+n \otimes 1$ ? If interpreted in the right way, is the resulting element normal? Does it satisfy the correct commutation relation with $u \otimes u$ ? Formally there are no problems with the last two questions but we are working with unbounded elements so we should tread carefully.

More precisely, we want the formal sum $u \otimes n+n \otimes 1$ (an operator whose domain is defined to be the intersection of the 
domain of both terms) to be closable and the closure will then be denoted by $u \otimes n \dot{+} n \otimes 1$. This last element has to be normal and should commute with $u \otimes u$ up to a factor $\nu$. But Woronowicz has proven in a fundamental paper $([75])$ that this is only possible if the spectrum of $n$ is in $C \cup\{0\}$, where

$$
C=\left\{\nu^{n} z \mid n \in \mathbb{Z} \text { and } z \in \mathbb{C} \text { with }|z|=1\right\}
$$

So $C$ is the union of all circles of which the radius is an integer power of $\nu$. This means that the generating relations mentioned above are not sufficient. We have to add the extra spectral relation that the spectrum of $n$ should be contained in $C \cup\{0\}$. This is truly a quantum phenomenon!

What are the solutions to these problems? It turns out that it is not so difficult to define the $\mathrm{C}^{*}$-algebra $A\left(E_{\nu}(2)\right)$. Let $\alpha$ be the action of $\mathbb{Z}$ on $C_{0}(C)$ such that $\alpha_{m}(f)(x)=f\left(\nu^{-m} x\right)$ for all $n \in \mathbb{Z}, x \in C$ and $f \in C_{0}(C)$. Define $A\left(E_{\nu}(2)\right)$ to be the crossed product $\mathrm{C}^{*}$-algebra $C_{0}(C) \times_{\alpha} \mathbb{Z}$. The element $n$ is the image of the identity function $\iota_{C}$ under the embedding of $C_{0}(C)$ in $A\left(E_{\nu}(2)\right)$, and the element $u$ is the image of 1 under the embedding of $\mathbb{Z}$ in $A\left(E_{\nu}(2)\right)$. By taking the crossed product of $\mathbb{Z}$ with $C_{0}(C)$ (rather than using the whole complex plane), the spectrum of $n$ is equal to $C \cup\{0\}$ (because this spectrum equals the spectrum of $\iota_{C}$, which is the closure of its image).

Implementing a co-multiplication as described in problem 2 above is possible, but it involves a lot of nontrivial unbounded operator theory. Guessing the formula for the left-invariant weight is very easy but it takes hard work to prove that it is left-invariant.

\section{References}

[1] E. ABe, Hopf Algebras. Cambridge Tracts in Mathematics 74. Cambridge University Press: Cambridge, 1980.

[2] V. I. Arnol'D, Mathematical Methods of Classical Mechanics. Springer-Verlag: New York, 1978.

[3] S. BAAJ, Représentation régulière du groupe quantique des déplacements de Woronowicz, Astérisque 232 (1995), 11-48. 
[4] S. BaAj \& G. Skandalis, Unitaires multiplicatifs et dualité pour les produits croisés de $C^{*}$-algèbres, Ann. Scient. Éc. Norm. Sup. 26 (1993) 425-488.

[5] T. Banica, Le groupe quantique compact libre $U(n)$, Commun. Math. Phys. 190 (1997), 143-172.

[6] A. Bauval, Quantum group and Poisson-deformation of $S U(2)$, preprint (1992).

[7] A. Connes, Noncommutative Geometry. Academic Press, 1994.

[8] P. A. M. Dirac, The Principles of Quantum Mechanics. Oxford University Press: Oxford, 1967.

[9] S. Doplicher, R. HaAg and J. E. Roberts, Local observables and particle statistics, I, Commun. Math. Phys. 23 (1971), 199-230.

[10] S. Doplicher and J. E. Roberts, A new duality theory for compact groups, Invent. Math. 98 (1989), 157-218.

[11] V. G. Drinfeld, Quantum groups, Proceedings ICM Berkeley (1986), 798-820.

[12] V. G. Drinfeld, Hamiltonian structures on Lie groups, Lie bialgebras and the geometric meaning of the classical Yang-Baxter equations, Soviet Math. Dokl. 27 (1983), 285-287.

[13] E. Effros \& Z.-J. RuAn, Discrete quantum groups I: The Haar measure, Int. J. Math. 5 (1994), 681-723.

[14] M. Enock \& J.-M. SchwarTz, Kac Algebras and Duality of Locally Compact Groups. Springer-Verlag: Berlin, 1992.

[15] M. Enock \& L. Vainerman, Deformation of a Kac algebra by an abelian subgroup, Comm. Math. Phys. 178 (1996), 571-596.

[16] M. Enock \& J.-M. VAllin, $C^{*}$-algèbres de Kac et algèbres de Kac, Proc. London Math. Soc. 66 (1993), 619-650.

[17] L. D. Faddeev, N. Yu. Reshetkhin and L. A. Takhtajan, Quantization of Lie groups and Lie algebras, Algebraic Analysis 1 (1988), 129-139.

[18] J. Fuchs, Affine Lie algebras and Quantum Groups. Cambridge Monographs on Mathematical Physics, 1992.

[19] H. Goldstein, Classical Mechanics. Addison Wesley, 1950.

[20] M. Jimbo, A q-difference analogue of $U(q)$ and the Yang-Baxter equation, Lett. Math. Phys. 10 (1985), 63-69.

[21] V. F. R. Jones, Notes on subfactors and statistical mechanics, International J. Modern Phys. A 5 (1990), 441-460.

[22] C. Kassel, Quantum Groups. Springer-Verlag: Berlin, 1995.

[23] D. Kazhdan \& G. Lusztig, Tensor structures arising from affine Lie algebras, I-IV, Journ. Am. Math. Soc. 6 (1993), 905-947, 6 (1993), 
949-1011, 7 (1994), 335-381, 7 (1994), 383-453.

[24] E. KirchBerG, Lecture at the conference 'Invariants in operator algebras', Copenhagen, August 1992.

[25] A. U. Klimyk \& K. Schmüdgen, Quantum Groups and Their Representations. Texts and Monographs in Physics. Springer Verlag, 1998.

[26] H. T. Koelink \& J. Verding, Spectral analysis and the Haar functional on the quantum $\mathrm{SU}(2)$ group, Commun. Math. Phys. 177 (1996), 399-415.

[27] J. Kustermans, Locally compact quantum groups in the universal setting, preprint, University College Cork (1999).

[28] J. Kustermans \& A. Van Daele, $C^{*}$-algebraic quantum groups arising from algebraic quantum groups, Int. J. Math. 8 (1997), 10671139.

[29] J. Kustermans \& L. Tuset, A survey of $C^{*}$-algebraic quantum groups, part I, Bull. Ir. Math. Soc. 43 (1999), 8-63.

[30] J. Kustermans \& S. VAas, Locally compact quantum groups, preprint, KU Leuven \& University College Cork (1999).

[31] J. Kustermans \& S. VAAs, Weight theory for $C^{*}$-algebraic quantum groups, preprint, KU Leuven \& University College Cork (1999).

[32] S. Levendorski And Y. Soibelman, Algebras of functions on compact quantum groups, Schubert cells and quantum tori, Commun. Math. Physics 139 (1991), 141-170.

[33] R. Longo, Index of subfactors and statistics of quantum fields, I, Commun. Math. Phys. 126 (1989), 217-247.

[34] R. Longo and J. E. Roberts, A theory of dimensions, K-Theory 11 (1997), 103-159.

[35] Y. Manin, Topics in Noncommutative Geometry. Princeton University Press, 1991.

[36] G. J. Murphy \& L. Tuset, Compact quantum groups, preprint (1999).

[37] T. Masuda \& Y. Nakagami, A von Neumann algebra framework for the duality of the quantum groups, Publ. Res. Inst. Math. Sci. 30 (1994), 799-850.

[38] T. Masuda, Y. Nakagami \& S. L. Woronowicz, Lectures at the Fields Institute and at the University of Warsaw, 1995.

[39] G. NAGY, On the Haar measure of the quantum $\mathrm{SU}(n)$ group, Comm. Math. Phys. 153 (1993), 217-228.

[40] G. K. Pedersen, C*-algebras and Their Automorphism Groups. Academic Press: London, 1979. 
[41] P. Podleś, Quantum spheres, Lett. Math. Phys. 14 (1987), 193202.

[42] P. Podleś \& S. L. Woronowicz, Quantum deformation of Lorentz group, Commun. Math. Phys. 130 (1990), 381-431.

[43] M. A. Rieffel, Compact quantum groups associated with toral subgroups, Contemp. Math. 145 (1993), 465-491.

[44] M. A. RIEffel, Deformation Quantization of Actions of $\mathbb{R}^{d}$. Memoirs A. M. S. 506. American Mathematical Society: Providence, 1993.

[45] M. A. Rieffel, Deformation quantization of Heisenberg manifolds, Commun. Math. Phys. 122 (1989), 531-562.

[46] M. A. Rieffel, Some solvable quantum groups, in Operator algebras and topology, 146-159. Proceedings of the OATE 2 conference, Bucharest, Romania, 1989, ed. W. B. Arveson et al. Pitman Research Notes in Mathematics 270, (1992).

[47] J. E. Roberts And L. Tuset, On the equality of q-dimension and intrinsic dimension, preprint, Universitá di Roma Tor Vergata (1998).

[48] M. Rosso, Comparaison des groupes SU(2) quantiques de Drinfeld et de Woronowicz, C. R. Acad. Sci. Paris Sér. I Math. 304 (1987), 323-326.

[49] K. Schmüdgen ANd A. Schuler, Classification of bicovariant differential calculi on quantum groups, Commun. Math. Phys. 170 (1995), 315-335.

[50] P. Schupp, P. Watts And B. Zumino, Bicovariant quantum algebras and quantum Lie algebras, Commun. Math. Phys. 157 (1993), 305-329.

[51] A. J-L. Sheu, Quantization of the Poisson SU(2) and its Poisson homogeneous space-the 2-sphere, Commun. Math. Phys. 135 (1991), 217-232.

[52] Y. SoIBELman, Irreducible representations of the algebra of functions on the quantum group $\mathrm{SU}(n)$ and Schubert cells. (Russian). Dokl. Akad. Nauk SSSR 307 (1989), 41-45.

[53] P. Stachura, Bicovariant differential calculi on $S_{\mu} U(2)$, Letters in Math. Physics 25 (1992), 175-188.

[54] M. Sugiura, Unitary Representations and Harmonic Aalysis. North Holland Mathematical Library, 1975.

[55] M. TAKesaki, Theory of Operator Algebras, I. Springer-Verlag: New York, 1979.

[56] L. Tuset, Differential Calculi on Quantum SU(2). Doctoral thesis, The Norwegian Institute of Technology (1995). 
[57] L. I. Vainerman \& G. I. KaC, Nonunimodular ring-groups and Hopf-von Neumann algebras, Math. USSR, Sbornik 23 (1974), 185214 .

[58] L. I. Vainerman \& G. I. KaC, Nonunimodular ring-groups and Hopf-von Neumann algebras, Soviet Math. Dokl. 14 (1973), 1144-1148. [59] L. L. Vaksman \& Y. S. Soibel'man, Algebra of functions on the quantum group SU(2), Funct. Anal. Appl. 22 (1988), 170-181.

[60] J.-M. VAllin, $C^{*}$-algèbres de Hopf et $C^{*}$-algèbres de Kac, Proc. London Math. Soc. 50 (1985), 131-174.

[61] A. VAn Daele, An algebraic framework for group duality, Adv. Math. 140 (1998), 323-366.

[62] A. Van Daele, Discrete quantum groups, J. Algebra 180 (1996), 431-444.

[63] A. VAn DAele, The Haar measure on a compact quantum group, Proc. Amer. Math. Soc. 123 (1995), 3125-3128.

[64] A. Van Daele, Multiplier Hopf Algebras, Trans. Amer. Math. Soc. 342 (1994), 917-932.

[65] A. VAn Daele \& S. L. Woronowicz, Duality for the quantum E(2) group, Pac. J. Math. 173 (1996), 375-385.

[66] S. L. VARAdARAJAn, Quantization and deformation, preprint (1991)

[67] S. L. Varadarajan, Lie Groups, Lie Algebras, and Their Representations. Springer-Verlag: New York, 1984.

[68] A. Wassermann, Operator algebras and conformal field theory, I \& III, Proc. ICM (1994) \& Invent. Math. 133 (1998), 467-538.

[69] H. WenzL, Hecke algebras of type $A_{n}$ and subfactors, Invent. Math. 92 (1988), 349-383.

[70] H. WenzL, Quantum groups and subfactors of type $B, C$ and $D$, Comm. Math. Phys. 133 (1990), 383-432.

[71] H. WenzL, $C^{*}$-tensor categories from quantum groups, Journ. Am. Math. Soc. 11 (1998), 261-282.

[72] S. L. Woronowicz, Compact quantum groups, in 'Symétries quantiques' (Les Houches, 1995), 845-884. North-Holland: Amsterdam, 1998.

[73] S. L. Woronowicz, From multiplicative unitaries to quantum groups, Int. J. Math. 7 (1996), 127-149.

[74] S. L. Woronowicz, Quantum E(2) group and its Pontryagin dual, Lett. Math. Phys. 23 (1991), 251-263.

[75] S. L. WORONOwicz, Unbounded elements affiliated with $C^{*}$-algebras and non-compact quantum groups, Commun. Math. Phys. 136 (1991), 399-432. 
[76] S. L. Woronowicz, Differential calculus on compact matrix pseudogroups (quantum groups), Commun. Math. Phys. 111 (1989), $125-170$.

[77] S. L. Woronowicz, Tannaka-Krein duality for compact matrix pseudogroups. Twisted SU( $N)$ groups, Invent. Math. 93 (1988), 35-76.

[78] S. L. Woronowicz, Compact matrix pseudogroups, Commun. Math. Phys. 111 (1987), 613-665.

[79] S. L. Woronowicz, Twisted SU(2) group. An example of a noncommutative differential calculus, Publ. Res. Inst. Math. Sci. 23 (1987), $117-181$

[80] S. L. Woronowicz, Pseudospaces, pseudogroups and Pontrjagin duality, in Proceedings of the International Conference on Mathematical Physics, Lausanne, 1979, pp.407-412. Lecture Notes in Physics 116 (1980).

Johan Kustermans

Department of Mathematics

University College Cork

Cork

Ireland

e-mail : johank@ucc.ie
Lars Tuset

Department of Mathematics University College Cork

Cork

Ireland

e-mail : lars.tuset@ucc.ie 